\title{
RECHERCHES SUR LES FONCTIONS ELLIPTIQUES
}

(par M. N. H. ABEL).

\section{SECOND MÉMOIRE.}

Dans les "recherches sur les fonctions elliptiques" insérées dans le tome second de ce journal j'ai fait voir comment on pourra toujours résoudre l'équation du degré $(2 n+1)^{2}$ d'où dépend la division en $2 n+1$ parties égales d'une fonction elliptique; mais je me suis contenté à démontrer seulement la possibilité d'une telle résolution, sans entrer dans des détails sur l'expression des racines. Une note de M. J AConI insérée dans le t. III, p. 86, m'a fait revenir sur cet objet; et étant parvenu à cette occasion à plusieurs propriétés nouvelles des fonctions elliptiques je vais continuer ici mes premières recherches.

$$
\$ 1 \text {. }
$$

En faisant $\varphi \theta=x$ on aura selon ce qu'on a vu dans le \& III du mémoire cité

$$
\varphi(2 n+1) \theta=R
$$

où $R$ une fonction rationnelle de $x$, le numérateur étant du degré $(2 n+\mathrm{I})^{2}$ et le dénominateur du degré $(2 n+1)^{2}-\mathrm{I}$. L'équation (I) est donc du degré $(2 n+1)^{2}$ et les racines pourront être représentées par la formule

$$
x=\varphi\left(\theta+\frac{2 m \omega+2 \mu \bar{\omega} i}{2 n+1}\right)
$$

en donnant à $m$ et $\mu$ toutes les valeurs entières depuis zéro jusqu’à $2 n$ incl. 
Soient pour abréger

$$
\frac{2(1)}{2 n+1}=\alpha, \quad \frac{2 \bar{w} i}{2 n+1}=\beta
$$

l'expression des racines sera:

$$
x=\varphi(\theta+m \alpha+\mu \beta) .
$$

Cela posé nous allons démontrer le théorème suivant:

Théorème I. Soit $\phi \theta$ une fonction entière quelconque des quantités $\varphi(\theta+m \alpha+\mu \beta)$ qui reste la même en changeant $\theta$ en $\theta+\alpha$ et $\theta+\beta$. Soit $\nu$ l'exposant le plus grand de la quantité $\varphi \theta$ dans la fonction $\phi \theta$; on aura toujours:

$$
\phi \theta=p+q \cdot f(2 n+\mathrm{I}) \theta \cdot F(2 n+1) \theta
$$

où $p$ et $q$ sont deux fonctions entières de $\varphi(2 n+1) \theta$; la première du degré $\nu$ et la seconde du degré $\nu-2$.

Démonstration. En vertu de la formule (10), pag. 105, on a

$$
\varphi(\theta+m \alpha+\mu \beta)=\frac{\varphi \theta \cdot f(m \alpha+\mu \beta) \cdot F(m \alpha+\mu \beta)+\varphi(m \alpha+\mu \beta) \cdot f \theta \cdot F \theta}{I+e^{z} c^{2} \cdot \varphi^{2}(m \alpha+\mu \beta) \cdot \varphi^{2} \theta}
$$

d'où il suit qu'on pourra exprimer $\phi \theta$ rationnellement en $\varphi \theta$ et $f \theta$. $\boldsymbol{E} \theta$. Or le carré de $f \theta . F \theta$ est rationnel en $\varphi \theta$, savoir

$$
(f \theta . F \theta)^{2}=\left(1-c^{2} \varphi^{2} \theta\right)\left(1+e^{2} \varphi^{2} \theta\right),
$$

donc on pourra faire en sorte que l'expression de $\psi \theta$ ne contienne la quantité $f \theta . F \theta$ qu'à la première puissance. On pourra donc faire:

$$
\psi \theta=\psi_{1}\{\varphi \theta\}+\phi_{2}\{\varphi \theta\} \cdot f \theta \cdot F \theta
$$

où $\psi_{1}\{\varphi \theta\}$ et $\psi_{2}\{\varphi \theta\}$ sont des fonctions rationnelles de $\varphi \theta$.

$\mathrm{Si}$ l'on met $\omega-\theta$ à la place de $\theta$ on aura en remarquant que $\varphi(\omega-\theta)=\varphi \theta ; f(\omega-\theta)=-f \theta ; F(\omega-\theta)=F \theta$ :

$$
\phi(\omega-\theta)=\phi_{1}\{\varphi \theta\}-\psi_{2}\{\varphi \theta\} . f \theta . F \theta .
$$


Des équations (7) et (8) on déduit:

$$
\begin{gathered}
\phi_{1}\{\varphi \theta\}=\frac{\mathrm{I}}{2} \cdot\{\phi \theta+\psi(\omega-\theta)\} \\
\psi_{2}\{\varphi \theta\} . f \theta . F \theta=\frac{\mathrm{I}}{2} \cdot\{\psi \theta-\psi(\omega-\theta\} .
\end{gathered}
$$

Considérons d'abord la fonction $\phi_{1}\{\varphi \theta\}$. En y mettant $\theta+\alpha$ au lieu de $\theta$ il viendra:

$$
\phi_{1}\{\varphi(\theta+\alpha)\}=\frac{\mathrm{I}}{2}\{\phi(\theta+\alpha)+\phi(\omega-\alpha-\theta)\}
$$

or on a $\phi(\theta+\alpha)=\phi \theta$ et par conséquent aussi en mettant $\omega-x-\theta$ au lieu de $\theta$

$$
\phi(\omega-\theta)=\psi(\omega-\alpha-\theta)
$$

done:

$$
\psi_{1}\{\varphi(\theta+a)\}=\frac{1}{2}\{\phi \theta+\phi(\omega-\theta)\}
$$

c'est-à-dire

$$
\varphi_{1}\{\varphi(\theta+\alpha)\}=\phi_{1}\{\varphi \theta\}
$$

On aura de la même manière:

$$
\psi_{1}\{\varphi(\theta+\beta)\}=\psi_{1}\{\varphi \theta\}
$$

La première de ces équations donne en mettant successivement $\theta+\alpha$, $\theta+2 \alpha, \ldots$ au lieu de $\theta$ :

$$
\phi_{1}\{\varphi(\theta+m \alpha)\}=\psi_{1}\{\varphi \theta\}
$$

où $m$ est un nombre entier quelconque. De même la seconde équation donne:

$$
\psi_{1}\{\varphi(\theta+\mu \beta)\}=\phi_{1}\{\varphi \theta\}
$$

d'où l'on tire en mettant $\theta+m a$ au lieu de $\theta$ et ayant égard à l'équation (1 1 ):

$$
\phi_{1}\{\varphi(\theta+m \alpha+\mu \beta)\}=\phi_{1}\{\varphi \theta\} .
$$

La fonction $\phi_{1}\{\varphi \theta\}$ reste donc la même en substituant au lieu de $\varphi \theta$ une autre racine quelconque de l'équation (1). La formule (1 2) donne 
en attribuant à $m$ et $\mu$ toutes les valeurs entières dépuis zéro jusqu'à $2 n$ et puis ajoutant:

$$
\left.\phi_{1}\{\varphi \theta\}=\frac{1}{(2 n+1)^{2}} \cdot \sum_{0}^{2 n} \sum_{0}^{2 n} \phi_{1}\{\theta+m \alpha+\mu \beta)\right\} .
$$

Le second membre de cette équation est une fonction rationnelle et symétrique des racines de l'équation ( $\mathrm{I}$ ), donc on pourra l'exprimer rationnellement en les coefficients de cette équation, cest-à-dire en $\varphi(2 n+1) \theta$. Soit donc

$$
\phi_{1}\{\varphi \theta\}=p .
$$

La quantité $p$ sera une fonction rationnelle de $\varphi(2 n+1) \theta$. Or je dis que $p$ doit toujours être entière. En effet soit $\varphi(2 n+1) \theta=y$ et $p=\frac{p^{\prime}}{q^{\prime}}$ où $p^{\prime}$ et $q^{\prime}$ sont des fonctions entières de $y$ sans diviseur commun. Soit $y=\varphi(2 n+1) o$ une racine de l'équation $q^{\prime}=0$; la quantité

$$
p^{\prime}=\frac{1}{2}\{\phi \theta+\phi(\omega-\theta)\}
$$

sera infinie en faisant $\theta=\hat{o}$, donc on doit avoir $\phi \hat{o}+\phi(\omega-\hat{o})=\frac{\mathrm{I}}{\mathrm{O}}$. Maintenant il est évident par la forme de la fonction $\psi \theta$ que cette équation ne peut pas subsister à moins qu'une quantité de la forme $\varphi(\hat{\partial}+m \alpha+\mu \beta)$ ou $\varphi(\omega-\hat{o}+m \alpha+\mu \beta)$ ait une valeur infinie. Soit donc $\varphi(\delta+m x+\mu \beta)=\frac{1}{0}$, on aura en vertu de l'équation (3o) pag. I I 3 :

$$
\grave{o}=\left(m^{\prime}+\frac{1}{2}\right) \omega+\left(n^{\prime}+\frac{1}{2}\right) \bar{\omega} i-m \alpha-\mu \beta
$$

où $m^{\prime}$ et $n^{\prime}$ sont des nombres entiers; or cette valeur de $o$ donne:

$$
=\varphi\left\{\left[(2 n+1) m^{\prime}+n-2 m\right] \omega+\left[(2 n+1) n^{\prime}+n-2 \mu\right] \bar{\omega} i+\frac{\omega}{2}+\frac{\bar{\omega}}{2} i\right\}
$$

c'est-à-dire ((26) pag. I I I $): \varphi(2 n+1) o=\frac{1}{0}$. Or cela est impossible car une racine quelconque de l'équation $q^{\prime}=0$ doit être finie. On trouvera de même que $\varphi(\omega-\partial+1 m \alpha+\mu \beta)$ donnera $\varphi(2 n+1) \partial=\frac{1}{0} . \quad$ La quantité $p$ est donc une fonction entière de $\varphi(2 n+1) \theta$. 
Considérons maintenant l'équation ( IO). En divisant les deux membres par $f(2 n+1) \boldsymbol{\theta} \cdot \boldsymbol{F}(2 n+\mathrm{I}) \boldsymbol{\theta}$ on aura:

$$
\frac{\phi_{2}\{\varphi \theta\} \cdot f \theta \cdot F \theta}{f(2 n+1) \theta \cdot F(2 n+1) \theta}=\frac{1}{2} \cdot \frac{\psi \theta-\psi(\omega-\theta)}{f(2 n+1) \theta \cdot F(2 n+1) \theta} .
$$

Comme on a vu (45) pag. I I 7 on aura

$$
f(2 n+\mathrm{I}) \theta=f \theta . u, \quad F(2 n+\mathrm{I}) \theta=F \theta . v
$$

où $u$ et $v$ sont des fonctions rationnelles de $\varphi \theta$; donc le second membre de l'équation précédente sera une fonction rationnelle de $\varphi \theta$. . En la désignant par $\chi\{\varphi \theta\}$ on aura:

$$
\chi\{\varphi \theta\}=\frac{1}{2} \cdot \frac{\varepsilon^{\prime} \theta-\psi(\omega-\theta)}{f(2 n+1) \theta \cdot F(2 n+1) \theta} .
$$

En mettant $\theta+\alpha$ au lieu de $\theta$, il viendra

$$
\begin{aligned}
\psi(\theta+\alpha) & =\phi \theta, \quad \psi(\omega-(\theta+\alpha))=\psi(\omega-\theta) \\
f(2 n+1)(\theta+\alpha) & =f((2 n+1) \theta+2 m \omega+2 \mu \bar{\omega} i)=f(2 n+1) \theta \\
F(2 n+1)(\theta+\alpha) & =F((2 n+1) \theta+2 m \omega+2 \mu \bar{\omega} i)=F(2 n+1) \theta
\end{aligned}
$$

donc on aura $\chi\{\varphi(\theta+\alpha)\}=\chi\{\varphi \theta\}$. De la même manière on trouvera $\chi\{\varphi(\theta+\beta)\}=\chi\{\varphi \theta\}$. On en déduit de même que plus haut pour la fonction $\psi_{1}\{\varphi \theta\}$ que $\chi\{\varphi \theta\}$ pourra être exprimée par une fonction entière de $\varphi(2 n+1) \theta$. Soit donc:

$$
\chi\{\varphi \theta\}=q
$$

on aura

$$
\psi_{2}\{\varphi \theta\} \cdot f \theta \cdot F \theta=q \cdot f(2 n+1) \theta \cdot F(2 n+1) \theta .
$$

Par conséquent enfin

$$
\phi \theta=p+q \cdot f(2 n+1) \theta \cdot F(2 n+1) \theta
$$

où $p$ et $q$ sont des fonctions entières de $\varphi(2 n+1) \theta$. Pour trouver les degrés de ces ionctions, soit $(\varphi \theta)^{\nu} \cdot \chi^{\theta}$ le terme dans $\phi \theta$ où $\varphi \theta$ est élevé à la plus haute puissance; on aura en posant $\varphi \theta$ infini

$$
\psi \theta=A \cdot(\varphi \theta)^{\nu}
$$


où $A$ est une constante. On aura de même

$$
\phi(\omega-\theta)=A^{\prime} \cdot(\varphi \theta)^{\nu}
$$

et par suite:

$$
p=\frac{\mathrm{I}}{2}\left(A+A^{\prime}\right)(\varphi \theta)^{\nu}
$$

mais pour $\varphi \theta$ infini on a $\varphi(2 n+1) \theta=B . \varphi \theta$ où $B$ est constante; d'où il suit que $p$ sera du degré $\nu$ par rapport à $\varphi(2 n+\mathrm{I}) \theta$. On démontrera de la même manière que la fonction $q$ sera du degré $\nu-2$ tout au plus. Notre théorème est donc démontré.

Dans le cas où la quantité $\varphi \theta$ ne monte qu'à la première puissance dans $\psi \theta$ on a $\nu=1$; par conséquent $q$ sera du degré $-\mathrm{I}$, c'est-à-dire $q=0$. On a donc alors:

$$
\phi \theta=A+B \cdot \varphi(2 n+\mathrm{I}) \theta
$$

où $A$ et $B$ sont des quantités constantes qu'on déterminera facilement en faisant $\theta=0$ et $\varphi \theta=\frac{1}{0}$.

Soit par ex.: $\pi \theta$ le produit d'un nombre quelconque des racines de l'équation ( $\mathrm{I}$ ) et faisons

$$
\phi \cdot \theta=\sum_{0}^{2 n} \sum_{0}^{2 n} \pi(\theta+m \alpha+\mu \beta)
$$

il est clair quon aura $\phi(\theta)=\psi(\theta+\alpha)=\psi(\theta+\beta)$ en remarquant que $\pi(\theta+(2 n+1) \alpha+\mu \beta)=\pi(\theta+\mu \beta)$ et $\pi(\theta+(2 n+1) \beta+m \alpha)=\pi(\theta+m \alpha)$.

Donc:

$$
\sum_{0}^{2 n} \sum_{n}^{2 n} \pi(\theta+m a+\mu \beta)=A+B \cdot \varphi(2 n+1) \theta .
$$

On doit remarquer que l'une des quantités $A$ et $B$ est toujours égale ì zéro. On a $A=\mathrm{o}$ si le nombre des facteurs de $\pi \theta$ est un nombre impair et $B=0$ si ce nombre est pair. Dans ce dernier cas la quantité $\phi \theta$ est donc indépendante de la valeur de $\theta$; par conséquent en faisant $\theta=0$ :

$$
\sum_{0}^{2 n} \sum_{0}^{2 n} \pi(\theta+m \alpha+\mu \beta)=\sum_{0}^{2 n} \sum_{n}^{2 n} \pi(m \alpha+\mu \beta) .
$$


Ainsi si l'on fait $\pi \theta=\varphi \theta \cdot \varphi\left(\theta+k \alpha+k^{\prime} \beta\right)$ on a:

$$
\begin{aligned}
& \sum_{0}^{2 n} \sum_{0}^{2 n} \varphi(\theta+m \alpha+\mu \beta) \cdot \varphi\left(\theta+(m+k) \alpha+\left(\mu+k^{\prime}\right) \beta\right) \\
= & \sum_{0}^{2 n} \sum_{0}^{2 n} \varphi(m \alpha+\mu \beta) \cdot \varphi\left((m+k) \alpha+\left(\mu+k^{\prime}\right) \beta\right),
\end{aligned}
$$

$k$ et $k^{\prime}$ sont des nombres entiers quelconques moindres que $2 n+\mathrm{I}$. Cependant on ne peut pas supposer à la fois $k=0 ; k^{\prime}=0$; car alors $\pi \theta=(\varphi \theta)^{2}$ et par suite $\nu=2$ tandis qu'on doit aroir $\nu=1$.

Entièrement de la même manière que nous avons démontré le théorème précédent on pourra encore établir les deux suivants:

Théorème II. Soit $\psi \theta$ une fonction quelconque entière des quantités de la forme $f(\theta+m \alpha+\mu \beta)$ telle que $\phi(\theta)=\phi(\theta+\alpha)=\phi(\theta+\beta)$, on aura:

$$
\phi \theta=p+q \cdot \varphi(2 n+\mathrm{I}) \theta \cdot F(2 n+\mathrm{I}) \theta
$$

où $p$ et $q$ sont des fonctions entières de $f(2 n+1) \theta$; la première du degré $\nu$ et la seconde du degré $\nu-2$ tout au plus, en clésignant par $\nu$ le plus grand exposant de $f \theta$ dans $\psi \theta$.

Théorème III. Soit $\phi \theta$ une fonction quelconque entière des quantités de la forme $F(\theta+m \alpha+\mu \beta)$ telle que $\phi \theta=\psi(\theta+\alpha)=\psi(\theta+\beta)$, on aura $\phi \theta=p+q \cdot \varphi(2 n+\mathrm{I}) \theta \cdot f(2 n+\mathrm{I}) \theta$, où $p$ et $q$ sont des fonctions entières de $F(2 n+$ I $) \theta$; la première du degre $\nu$ et la seconde du degré $\nu-2$ tout au plus, en désignant par $\nu$ le plus grand exposant de $F \theta$ dans $\phi \theta$.

\section{$\S 2$.}

A l'aide du théorème I, démontré dans le paragraphe précédent, il est facile de parvenir au théorème de $M$. $J_{A C O B I}$ sur la forme des racines de l'équation:

$$
\varphi(2 n+\mathrm{I}) \theta=R
$$

Considérons l'expression:

$$
\phi \theta=\sum_{0}^{2 n} \sum_{0}^{2 n} \delta^{m k+\jmath k^{\prime}} \cdot \varphi(\theta+m \alpha+\mu \beta)
$$

Acta mathematica, 26. Imprimé le 21 mars 1902. 
où $\quad o=\cos \frac{2 \pi}{2 n+\mathrm{I}}+i \cdot \sin \frac{2 \pi}{2 n+\mathrm{I}}$ et $k$ et $k^{\prime}$ sont deux nombres entiers. En mettant $\theta+\alpha$ au lieu de $\theta$, on aura

$$
\phi(\theta+\alpha)=\sum_{0}^{2 n} \delta^{\mu k^{\prime}} \cdot \sum_{0}^{2 n} \delta^{m k} \varphi(\theta+(m+1) \alpha+\mu \beta)
$$

or on a

mais

$$
\begin{aligned}
\sum_{0}^{2 n} \delta^{n k t} \varphi(\theta+ & (m+1) \alpha+\mu \beta)=\sum_{1}^{2 n} \delta^{(m-1) k} \varphi(\theta+m \alpha+\mu \beta) \\
& +\delta^{2 n k} \varphi(\theta+(2 n+1) \alpha+\mu \beta),
\end{aligned}
$$

$$
\varphi(\theta+\mu \beta+(2 n+1) \alpha)=\varphi(\theta+\mu \beta+2 \omega)=\varphi(\theta+\mu \beta),
$$

donc en remarquant que $\delta^{2 n k}=\delta^{-k}$ :

$$
\sum_{0}^{2 n} \delta^{m k} \varphi(\theta+(m+1) \alpha+\mu \beta)=o^{-k} \cdot \sum_{0}^{2 n} \varphi(\theta+m \alpha+\mu \beta) .
$$

L'expression de $\phi(\theta+\alpha)$ deviendra donc en substituant:

$$
\phi(\theta+a)=\delta^{-k} \cdot \phi \theta .
$$

Entièrement de la même manière on aura:

$$
\phi(\theta+\beta)=\delta^{-k^{\prime}} \cdot \phi \theta
$$

En élevant chaque membre de $(20)$ à la $(2 n+1)^{\mathrm{e}}$ puissance et remarquant que $\delta^{-k(2 n+1)}=\mathbf{I}=\hat{o}^{-k^{\prime}(2 n+1)}$ il viendra:

$$
\{\phi \theta\}^{2 n+1}=\{\phi(\theta+\alpha)\}^{2 n+1}=\{\phi(\theta+\beta)\}^{2 n+1} .
$$

D'où il suit que le théorème $\mathrm{I}^{\text {er }}$ est applicable à la fonction $(\psi \theta)^{2 n+1}$. On aura donc:

$$
(\psi \theta)^{2 n+1}=p+q \cdot f(2 n+1) \theta \cdot F(2 n+1) \theta
$$

où $p$ et $q$ sont des fonctions entières de $\varphi(2 n+1) \theta$.

L'expression (I9) de $\phi \theta$ nous montre que $\varphi \theta$ se trouve à la première puissance seulement dans cette fonction; donc $\varphi \theta$ est élevé à la puissance $2 n+\mathrm{I}$ dans la fonction $(\psi \theta)^{2 n+1}$. De là il suit que $p$ et $q$ seront respec- 
tivement du degré $2 n+1$ et $2 n-1$ tout au plus. Comme nous allons voir $g$ n'est effectivement que du degré $2 n-2$.

En mettant dans l'expression de $(\psi \theta)^{2 n+1} \omega-\theta$ au lieu de $\theta$ on aura en remarquant que

$$
\begin{gathered}
\varphi(2 n+1)(\omega-\theta)=\varphi(2 n+1) \theta \\
f(2 n+1)(\omega-\theta)=-f(2 n+1) \theta, \quad F(2 n+1)(\omega-\theta)=F(2 n+1) \theta
\end{gathered}
$$

cette formule:

$$
\{\phi(\omega-\theta)\}^{2 n+1}=p-q \cdot f(2 n+1) \theta \cdot F(2 n+1) \theta
$$

done:

$$
\left\{\begin{array}{c}
2 p=(\phi \theta)^{2 n+1}+\{\phi(\omega-\theta)\}^{2 n+1} \\
2 q \cdot f(2 n+1) \theta \cdot F(2 n+1) \theta=(\phi \theta)^{2 n+1}-\{\phi(\omega-\theta)\}^{2 n+1} .
\end{array}\right.
$$

Mettons ici - $\theta$ au lieu de $\theta$ et désignons les valeurs correspondantes de $p$ et $q$ par $p^{\prime}$ et $q^{\prime}$, on aura, comme il est facile de déduire de la formule (19),

$$
\phi(-\theta)=-\phi(\omega-\theta) ; \quad \psi(\omega+\theta)=-\phi \theta
$$

donc

$$
p^{\prime}=-p, \quad q^{\prime}=q .
$$

Maintenant puisque $\varphi(2 n+1) \theta$ change de signe avec $\theta$, il s'ensuit que $p$ ne contiendra que des puissances impaires et $q$ seulement des puissances paires. On aura donc

$$
\left\{\begin{array}{r}
p=\left(a_{0}+a_{1}\{\varphi(2 n+1) \theta\}^{2}+a_{2}\{\varphi(2 n+1) \theta\}^{4}+\ldots\right. \\
\left.+a_{n}\{\varphi(2 n+1) \theta\}^{2 n}\right) \cdot \varphi(2 n+\mathrm{I}) \theta \\
q=b_{0}+b_{1}\{\varphi(2 n+1) \theta\}^{2}+b_{2}\{\varphi(2 n+1) \theta\}^{4}+\ldots \\
+b_{n-1} \cdot\{\varphi(2 n+1) \theta\}^{2 n-2}
\end{array}\right.
$$

où $a_{0}, a_{1}, \ldots, a_{n} ; b_{0}, b_{1}, \ldots, b_{n-1}$ sont des coefficients indépendants de $\theta$. Ces coefficients sont des fonctions des quantités $\varphi(m \alpha+\mu \beta)$ et on pourra les déterminer en développant les deux membres de l'équation (22) suivant les puissances de $\varphi \theta$. Or le procédé serait fort compliqué. Nous donnerons tout à l'heure une autre méthode plus simple. 
Connaissant ainsi la valeur de $(\psi \theta)^{2 n+1}$ en $\varphi(2 n+1) \theta$, on en tire celle de $\phi \theta$ en extrayant la racine $(2 n+1)^{\prime \prime}$ savoir

$$
\psi \theta=\sqrt[2 n+1]{1+q \cdot f(2 n+\mathrm{I}) \theta \cdot F(2 n+\mathrm{I}) \boldsymbol{\theta}},
$$

$\phi \theta$ est une fonction linéaire des racines. Elle prend différentes formes selon les valeurs différentes de $k$ et $k^{\prime}$. En donnant ì ces nombres toutes les valeurs depuis zéro jusqu'a $2 n$ on connait la valeur de $(2 n+1)^{2}$ fonctions différentes. Nous allons voir qu'on pourra en déduire les racines elles-mêmes.

Désignons la valeur de $p+q f(2 n+1) \theta \cdot F(2 n+1) \theta$ qui répond à $k$ et $k^{\prime}$ par $\chi\left(k, k^{\prime}\right)$, on aura:

$$
\sum_{0}^{2 n} \sum_{0}^{2 n}{ }_{0} \delta^{m k+\mu k^{\prime}} \cdot \varphi(\theta+m \alpha+\mu \beta)=\sqrt[2 n+1]{\chi} \frac{\left(k, k^{\prime}\right)}{} .
$$

Multiplions cette équation par $\delta^{-m^{\prime} k-y^{\prime} k^{\prime}}$ et prenons ensuite la somme par rapport à $k$ et $k^{\prime}$ depuis zéro jusqu'à $2 n$, on aura:

$$
\sum_{0}^{2 n} \sum_{0}^{2 n} \sum_{0}^{2 n} \sum_{0}^{2 n} \sum_{k^{\prime}} \hat{\delta}^{\left(m-m^{\prime}\right) k+\left(\mu-k^{\prime}\right) k^{\prime}} \cdot \varphi(\theta+m \alpha+\mu \beta)=\sum_{0}^{2 n} \sum_{0}^{2 n} \sqrt[2 n+1]{k^{\prime}} \sqrt{\chi^{\left(k, k^{\prime}\right)}} \cdot \partial^{-m i^{\prime} k-\mu^{\prime} k^{\prime}} .
$$

Maintenant on a :

$$
\begin{gathered}
\sum_{0}^{2 n} \sum_{0}^{2 n} k^{\prime} \delta^{\left(m-m^{\prime}\right) k+\left(\mu-\mu^{\prime}\right) k^{\prime}}=\sum_{0}^{2 n} \delta^{\left(m-m^{\prime}\right) k} \cdot \sum_{0}^{2 n}{k^{\prime}}^{\left(\mu-\mu^{\prime}\right) k^{\prime}} \\
=\left\{\mathrm{I}+\delta^{m-m^{\prime}}+\delta^{2\left(m-m^{\prime}\right)}+\ldots+\delta^{2 n\left(m-m^{\prime}\right)}\right\} \cdot\left\{\mathrm{I}+\delta^{\prime n-\mu^{\prime}}+\delta^{2\left(\mu-\mu^{\prime}\right)}+\ldots+o^{2 n\left(u-\mu^{\prime}\right)}\right\}
\end{gathered}
$$

Quel que soit le nombre impair $2 n+$ I premier ou non, si en même temps $m^{\prime}$ et $\mu^{\prime}$ sont positifs et moindres que $2 n+$ I cette quantité se réduit à zéro pour toutes les valeurs de $m, m^{\prime}, \mu, \mu^{\prime}$ excepté lorsqu'on a à la fois $m==m^{\prime}$ et $\mu=\mu^{\prime}$; dans ce cas elle devient $(2 n+1)^{2}$. De là il suit que le premier membre de l'équation (26) se réduira à

$$
(2 n+1)^{2} \cdot \varphi\left(\theta+m^{\prime} \alpha+\mu^{\prime} \beta\right)
$$

Par conséquent on aura en changeant $m^{\prime}$ et $\mu^{\prime}$ en $m$ et $\mu$

$$
\varphi(\theta+m \alpha+\mu \beta)=\frac{\mathrm{I}}{(2 n+1)^{2}} \sum_{0}^{2 n} \sum_{0}^{2 n} k^{\prime} \partial^{-m k-\mu k^{\prime}}: \sqrt[2 n+1]{\chi\left(k, k^{\prime}\right)} ;
$$


voici donc l'expression d'une racine quelconque de l'équation

$$
\varphi(2 n+\mathrm{I}) \theta-R=\mathrm{o} .
$$

En faisant $m=0, \mu=0$ on aura la valeur de $\varphi \theta$ savoir

$$
\varphi \theta=\frac{1}{(2 n+1)^{2}} \cdot \sum_{0}^{2 n} \sum_{0}^{2 n} \sqrt[2 n+1]{\chi^{\prime\left(k, k^{\prime}\right)}} .
$$

L'expression des racines contient comme on voit $(2 n+1)^{2}$ radicaux différens. Or on peut les exprimer rationnellement en deux d'entre eux. Soient $\rho$ et $\rho_{1}$ les deux valeurs de $\chi_{\left(k, k^{\prime}\right)}$ qui répondent respectivement à $k=\mathrm{I}, k^{\prime}=\mathrm{o}$ et $k=\mathrm{o}, k^{\prime}=\mathrm{I}$, en sorte que:

$$
\left\{\begin{array}{l}
\rho=\left\{\sum_{0}^{2 n} \sum_{0}^{2 n} \delta^{m} \cdot \varphi(\theta+m \alpha+\mu \beta)\right\}^{2 n+1}, \\
\rho_{1}=\left\{\sum_{0}^{2 n} \sum_{0}^{2 n} \partial^{\mu} \cdot \varphi(\theta+m \alpha+\mu \beta)\right\}^{2 n+1},
\end{array}\right.
$$

alors je dis qu'on aura:

$$
\phi \theta=S \cdot \rho^{-1+\frac{k}{2 n+1}} \cdot \rho_{1}^{-1+\frac{k^{\prime}}{2 n+1}}
$$

où $S$ désigne une fonction entière de $\varphi(2 n+\mathrm{I}) \theta$ et $f(2 n+\mathrm{I}) \boldsymbol{\theta} \cdot \boldsymbol{F}(2 n+\mathrm{I}) \boldsymbol{\theta}$. Soient $\sqrt[2 n+1]{\rho}-\bar{c}=\phi_{1} \theta, \sqrt[2 n+1]{\rho_{1}}=\psi_{2} \theta$, on aura en vertu des équations (20), (2 I ) en faisant $k=\mathrm{I}, k^{\prime}=0 ; k=0, k^{\prime}=\mathrm{I}$

$$
\begin{aligned}
\phi_{1}(\theta+\alpha)=o^{-1} \cdot \phi_{1} \theta ; & \phi_{2}(\theta+\beta)=o^{-1} \cdot \phi_{2} \theta \\
\phi_{1}(\theta+\beta)=\phi_{1} \theta ; & \phi_{2}(\theta+\alpha)=\phi_{2} \theta
\end{aligned}
$$

donc:

$$
\begin{aligned}
& \left\{\psi_{1}(\theta+\alpha)\right\}^{-k+2 n+1}=\delta^{+k} \cdot\left(\psi_{1} \theta\right)^{-k+2 n+1}, \\
& \left\{\psi_{2}(\theta+\beta)\right\}^{-k^{\prime}+2 n+1}=\delta^{+k^{\prime}} \cdot\left(\psi_{2} \theta\right)^{-k^{\prime}+2 n+1},
\end{aligned}
$$

et de là en ayant égard à la formule (20)

$$
\begin{aligned}
& \psi(\theta+\alpha) \cdot\left\{\phi_{1}(\theta+\alpha)\right\}^{2 n+1-k} \cdot\left\{\psi_{2}(\theta+\alpha)\right\}^{2 n+1-k^{\prime}}=\psi \theta\left(\psi_{1} \theta\right)^{2 n+1-k} \cdot\left(\phi_{2} \theta\right)^{2 n+1-k^{\prime}}, \\
& \phi(\theta+\beta) \cdot\left\{\phi_{1}(\theta+\beta)\right\}^{2 n+1-k} \cdot\left\{\phi_{2}(\theta+\beta)\right\}^{2 n+1-k^{\prime}}=\psi \theta\left(\phi_{1} \theta\right)^{2 n+1-k} \cdot\left(\phi_{2} \theta\right)^{2 n+1-k^{\prime}},
\end{aligned}
$$

d'où il suit en vertu du théorème premier qu'on peut faire:

$$
\phi^{\prime} \theta \cdot\left(\phi_{1} \theta\right)^{2 n+1-k} \cdot\left(\psi_{2} \theta\right)^{2 n+1-k^{\prime}}=u+v \cdot f(2 n+1) \theta \cdot F(2 n+\mathrm{I}) \theta=S
$$


où $u$ et $v$ sont des fonctions entières de $\varphi(2 n+1) \theta$ et par suite

$$
\phi \theta=\left(u+v \cdot f(2 n+1) \theta \cdot F^{\prime}(2 n+1) \theta\right) \cdot \frac{\frac{\rho^{\frac{k}{2 n+1}} \cdot \rho_{t}^{\frac{k^{\prime}}{2 n+1}}}{\rho \cdot \rho_{1}}}{\rho+\sqrt[2 n+1]{\chi^{\left(k, k^{\prime}\right)}}}
$$

En vertu de cette formule le second membre de l'équation (27) deviendra une fonction rationnelle de $\varphi(2 n+1) \theta, f(2 n+1) \theta \cdot F(2 n+1) \theta, \sqrt[2 n+1]{\rho}$ et $\sqrt[2 n+1]{\rho_{1}}$ et cette fonction satisfait à l'équation ( $\mathrm{r}$ ) en donnant aux radicaux $\sqrt[2 n+1]{\rho}$ et $\sqrt[2+1]{\rho_{1}}$ tontes leurs valeurs.

Revenons maintenant à la détermination des fonctions $p$ et $\dot{q}$ dans la formule (22). Faisons $\theta=\frac{\varepsilon}{2 n+1}$, nous aurons:

$$
\begin{aligned}
& \left\{\phi\left(\frac{\varepsilon}{2 n+1}\right)\right\}^{2 n+1}=a_{0} \varphi \varepsilon+a_{1}(\varphi \varepsilon)^{3}+\ldots+a_{n}(\varphi \varepsilon)^{2 n+1} \\
& +\left\{b_{0}+b_{1}(\varphi \varepsilon)^{2}+\ldots+b_{n-1}(\varphi \varepsilon)^{2 n-2}\right\} \cdot f \varepsilon \cdot F \varepsilon .
\end{aligned}
$$

Cela posé supposons qu'on donne à $\varepsilon$ une telle valeur que $\phi\left(\frac{\varepsilon}{2 n+1}\right)=0$, on aura l'équation:

$$
\begin{gathered}
0=a_{0} \varphi \varepsilon+a_{1}(\varphi \varepsilon)^{3}+\ldots+a_{n}(\varphi \varepsilon)^{2 n+1} \\
+\left\{b_{0}+b_{1}(\varphi \varepsilon)^{2}+\ldots+b_{n-1}(\varphi \varepsilon)^{2 n-1}\right\} \cdot f \varepsilon . F \varepsilon
\end{gathered}
$$

que nous représenterons par $v=0$.

Maintenant il est clair d'après la forme du premier membre de l'équation (32) que l'équation $v=0$ aura encore lieu en la différentiant par rapport à $\varepsilon$ un nombre quelconque de fois moindre que $2 n+1$. On obtiendra de cette manière ces $2 n+1$ équations:

$$
v=0, \quad \frac{\partial v}{\partial \varepsilon}=0, \quad \frac{\partial^{2} v}{\partial \varepsilon^{2}}=0, \quad \ldots, \quad \frac{\partial^{2 n} v}{\partial \varepsilon^{2 n}}=0
$$

qui sont toutes linéaires par rapport aux inconnues $a_{0}, a_{1}, \ldots, a_{n}, b_{0}, b_{1}, \ldots$, $b_{n-1}$. En supposant $\varepsilon$ connu ces équations donneront les coefficients précédents en l'un d'entre eux; il faut donc encore une équation. Or si l'on divise les deux membres de l'équation par $(\varphi \theta)^{2 n+1}$ et qu'on suppose ensuite 
$\varphi \theta=\frac{\mathrm{I}}{\mathrm{o}}$ le premier membre se réduit à $\mathrm{I}$ et le second à $a_{n} \frac{\mathrm{I}}{(2 n+1)^{2 n+1}}$ à cause de la formule $\frac{\varphi(2 n+\mathrm{I}) \theta}{\varphi \theta}=\frac{\mathrm{I}}{2 n+\mathrm{I}}$ pour $\varphi \theta=\frac{\mathrm{I}}{\mathrm{o}}$. On aura donc:

$$
a_{n}=(2 n+1)^{2 n+1} \text {. }
$$

Les mêmes équations (35) donneront encore une équation en $\varphi \varepsilon$ seul; mais comme elle doit avoir lieu quelle que soit la valeur de $k$ et $k_{1}$ il sera impossible d'en tirer la valeur de $\varphi \varepsilon$ qui doit avoir effectivement lieu. Or on peut trouver cette quantité de la manière suivante: Soit

$$
\pi \theta=\phi \theta \cdot \phi(-\theta)
$$

on aura

$$
\pi(\theta+\alpha)=\phi(\theta+\alpha) \cdot \psi(-\theta-\alpha),
$$

mais selon $(20)$ on a $\phi(\theta+\alpha)=\partial^{-k} \cdot \phi \theta$ et de là en mettant - $\theta-\alpha$ au lieu de $\theta \phi(-\theta-a)=\delta^{k} \cdot \psi(-\theta)$ donc en substituant $\pi(\theta+\alpha)=\pi \theta$. De la même manière on prouvera que $\pi(\theta+\beta)=\pi \theta$. Donc en vertu du $I^{\text {er }}$ théorème

$$
\begin{aligned}
\psi \theta \cdot \psi(-\theta) & =A+B \cdot \varphi(2 n+\mathrm{I}) \theta+C \cdot\{\varphi(2 n+\mathrm{I}) \theta\}^{2} \\
& +D \cdot f(2 n+\mathrm{I}) \theta \cdot F(2 n+\mathrm{I}) \theta .
\end{aligned}
$$

Si l'on met $\omega-\theta$ au lieu de $\theta$ le premier membre reste le même et le second reste aussi le même en changeant seulement le signe de $D$. Ce coefficient est donc égal à zéro. En remarquant de plus que le second membre doit rester invariable en changeant le signe de $\theta$ et celui de $\varphi(2 n+\mathrm{I}) \theta$, on aura encore $B=\mathrm{o}$ et par conséquent

$$
\phi \theta \cdot \phi(-\theta)=A+C \cdot\{\varphi(2 n+1) \theta\}^{2} .
$$

En faisant $\theta=0$ on aura $A=\{\phi(0)\}^{2}$; en faisant $\varphi \theta=\frac{\mathrm{I}}{\mathrm{o}}$ on aura $C=-(2 n+1)^{2}$ et en faisant $\theta=\frac{\varepsilon}{2 n+1}, \psi \theta=0$ et $A+C \cdot(\varphi \varepsilon)^{2}=0$; on aura donc:

$$
\left\{\begin{array}{c}
\phi \theta \cdot \phi(-\theta)=(2 n+1)^{2}\left\{(\varphi s)^{2}-\{\varphi(2 n+1) \theta\}^{2}\right\} \text { et } \\
\varphi \varepsilon= \pm \frac{1}{2 n+1} \cdot \sum_{0}^{2 n} \sum_{0}^{2 n} \partial^{m k+n k^{\prime}} \cdot \varphi(m \alpha+\mu \beta) .
\end{array}\right.
$$


On connait donc $\varphi \varepsilon$ au signe près, or ce-ci n'influe en rien sur la valeur des coefficients $a_{0}, a_{1}, \ldots, b_{0}, b_{1}, \ldots$ En effet il n'est pas difficile de voir qu'on peut les exprimer rationnellement en $(\varphi s)^{2}$.

Le coefficient $b_{0}$ se trouve immédiatement en faisant $\varepsilon=0$ savoir

$$
b_{0}=\left\{\sum_{0}^{2 n} \sum_{0}^{2 n} \delta^{m k+\mu k^{\prime}} \cdot \varphi(m \alpha+\mu \beta)\right\}^{2 n+1}= \pm(2 n+1)^{2 n+1} \cdot(\varphi \varepsilon)^{2 n+1} .
$$

Les fonctions $p$ et $q$ jouissent d'une propriété remarquable qu'on peut déduire sur le champ de l'équation (38). En effet en élevant les deux membres à la $(2 n+\mathrm{I})^{\mathrm{e}}$ puissance on aura

$$
\{\phi \theta\}^{2 n+1} \cdot\{\phi(-\theta)\}^{2 n+1}=(2 n+1)^{4 n+2}\left\{(\varphi \varepsilon)^{2}-\{\varphi(2 n+1) \theta\}^{2}\right\}^{2 n+1}
$$

mais

$$
\begin{aligned}
\{\phi \theta\}^{2 n+1} & =p+q \cdot f(2 n+\mathrm{I}) \theta \cdot F(2 n+\mathrm{I}) \theta \\
\{\phi(-\theta)\}^{2 n+1} & =-p+q \cdot f(2 n+\mathrm{I}) \theta \cdot F(2 n+\mathrm{I}) \theta
\end{aligned}
$$

done en substituant

$$
\begin{gathered}
p^{2}-q^{2} \cdot\{f(2 n+1) \theta \cdot F(2 n+\mathrm{I}) \theta\}^{2} \\
=(2 n+1)^{4 n+2}\left\{\{\varphi(2 n+1) \theta\}^{2}-(\varsigma \varepsilon)^{2}\right\}^{2 n+1} .
\end{gathered}
$$

Soit $\varphi(2 n+1) \theta=y$ on aura

done

$$
\{f(2 n+\mathrm{I}) \theta \cdot F(2 n+\mathrm{I}) \theta\}^{2}=\left(\mathrm{I}-c^{2} y^{2}\right)\left(\mathrm{I}+e^{2} y^{2}\right)
$$

$$
\begin{aligned}
& \left(a_{0} y+a_{1} y^{3}+\ldots+a_{n} y^{2 n+1}\right)^{2} \\
& -\left(b_{0}+b_{1} y^{2}+\ldots+b_{n-1} y^{2 n-2}\right)^{2}\left(\mathrm{I}-c^{2} y^{2}\right)\left(\mathrm{I}+e^{2} y^{2}\right) \\
& =(2 n+\mathrm{I})^{4 n+2} \cdot\left\{y^{2}-\varphi^{2} \varepsilon\right\}^{2 n+1}
\end{aligned}
$$

Cette propriété des fonctions $p$ et $q$ suffit pour les déterminer au signe près car l'équation doit aroir lieu pour une valeur quelconque de $y$, et donnera ainsi $2 n+2$ équations entre les coefficients $a_{0}, a_{1}, \ldots, b_{0}, b_{1}, \ldots$ et $(\varphi \varepsilon)^{2}$.

Pour donner un exemple, considérons le cas le plus simple où $n=1$.

Dans ce cas on a $\alpha=\frac{2 \omega}{3}, \beta=\frac{2 \bar{\omega} i}{3}$

$$
\psi \theta=\sum_{0}^{2} \sum_{0}^{2} i^{m k+\mu k} \cdot \varphi\left(\theta+\frac{2 m \omega+2 \mu \bar{\omega} i}{3}\right)
$$


ou bien

$$
\begin{aligned}
& \phi \theta=\varphi \theta+\delta^{k} \cdot \varphi\left(\theta+\frac{2 \omega}{3}\right)+\delta^{2 k} \cdot \varphi\left(\theta+\frac{4 \omega}{3}\right)
\end{aligned}
$$

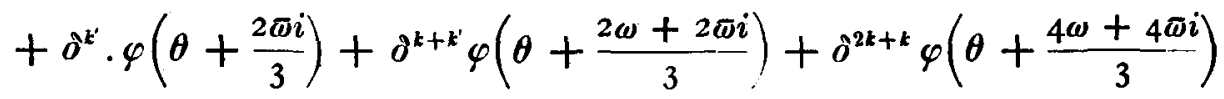

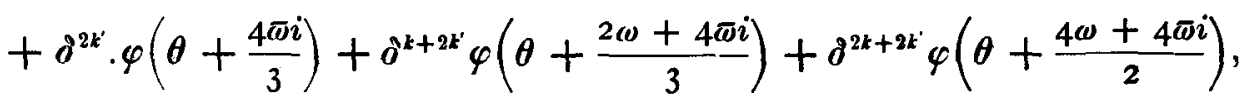

$$
\begin{aligned}
& \{\varphi \theta\}^{3}=a_{0} \varphi_{3} \theta+a_{1}\left(\varphi_{3} \theta\right)^{2}+b_{0} \cdot f_{3} \theta . F_{3} \theta \\
& \left(a_{0} y+a_{1} y^{3}\right)^{2}-b_{0}^{2}\left(1-c^{2} y^{2}\right)\left(1+e^{2} y^{2}\right)=3^{6} \cdot\left(y^{2}-f^{2}\right)^{3}
\end{aligned}
$$

où

$$
\cdot f=\frac{\mathrm{I}}{3} \cdot \sum_{0}^{3} \sum_{0}^{2} \partial^{m k+\mu k^{\prime}} \varphi\left\{\frac{2 m \omega+2 \mu \bar{\omega} i}{3}\right\} .
$$

En égalant les coefficients de $y^{4}$ dans les deux membres il viendra:

$$
2 a_{0} a_{1}+b_{0}^{2} c^{2} e^{2}=-3^{7} f^{2}
$$

maintenant

$$
a_{1}=3^{3} ; \quad b_{0}=3^{3} f^{3}
$$

donc

$$
a_{0}=-\frac{1}{2}\left\{3 f^{2}+e^{2} c^{2} f^{6}\right\} \cdot 3^{3}
$$

on a par suite

$$
\{\phi \theta\}^{3}=3^{3} \cdot\left\{(\varphi 3 \theta)^{3}-\frac{1}{2}\left(3 f^{2}+e^{2} c^{2} f^{6}\right) \cdot \varphi 3 \theta+f^{3} \cdot f 3 \theta \cdot F(3 \theta)\right\}=\chi\left(k, k^{\prime}\right)
$$

et de là

$$
\psi \theta=3 \cdot \sqrt[3]{\left(\varphi_{3} \theta\right)^{3}-\frac{1}{2}\left(3 f^{2}+e^{2} c^{2} f^{6}\right) \varphi_{3} \theta+f^{3} \cdot f_{3} \theta \cdot F_{3} \theta}
$$

La quantité $f$ est donnée par l'équation (42). Si l'on la cherche à l'aide de l'équation $\left(4 I^{\prime}\right)$ on parviendra à une équation du huitième degré. En effet en comparant les coefficients de $y^{2}$ on aura

$$
a_{0}^{2}+b_{0}^{2}\left(c^{2}-e^{2}\right)=3^{7} \cdot f^{4}
$$

c'est à dire en substituant les valeurs de $a_{0}$ et $b_{0}$

$$
\frac{1}{4} \cdot 3^{6} \cdot\left(3 f^{2}+e^{2} c^{2} f^{6}\right)^{2}+3^{6} \cdot\left(c^{2}-e^{2}\right) f^{6}-3^{7} \cdot f^{4}=0
$$


ou bien en développant:

$$
f^{8}+6 e^{2} c^{2} f^{4}+4\left(c^{2}-e^{2}\right) \cdot f^{2}-3=0 .
$$

Les huit racines de cette équation sont les huit valeurs de $f$ qu'on obtiendra, en donnant à $k$ et $k^{\prime}$ les valeurs $0,1,2$ en faisant abstraction de la valeur $f=0$ qui répond à $k=0$ et $k^{\prime}=0$.

Rien n'a été plus facile que de déterminer la valeur de $\phi \theta$ dans le cas où $n=\mathrm{J}$, mais si $n$ est plus grand il est très compliqué de se servir de la méthode exposée; c'est pourquoi nous allons exposer une autre qui conduira à l'expression générale et explicite de la fonction $\psi \theta$.

Désignons par $\pi(k)$ et $\pi_{1}\left(k^{\prime}\right)$ les valeurs de la fonction $\phi \theta$, qui répondent respectivement à $k^{\prime}=0$ et $k=0$, nous aurons:

$$
\left\{\begin{array}{l}
\pi(k)=\sum_{0}^{2 n} \sum_{0}^{2 n} \delta^{m k} \varphi(\theta+m \alpha+\mu \beta) ; \\
\pi_{1}\left(k^{\prime}\right)=\sum_{0}^{2 n} \sum_{0}^{2 n}{ }_{0} \delta^{\mu k^{\prime}} \varphi(\theta+m \alpha+\mu \beta) .
\end{array}\right.
$$

Soient de même $\pi^{\prime}(k)$ et $\pi_{1}^{\prime}\left(k^{\prime}\right)$ les valeurs de $\pi(k)$ et $\pi_{1}\left(k^{\prime}\right)$ en mettant - $\theta$ au lieu de $\theta$.

Cela posé considérons la fonction

$$
P=\pi(k) \cdot \pi_{1}\left(k^{\prime}\right) \cdot \phi(-\theta) .
$$

En y mettant $\theta+\alpha$ au lieu de $\theta$ les fonctions

$$
\pi(k) ; \pi_{1}\left(k^{\prime}\right) ; \phi(-\theta)
$$

deviendront respectivement

$$
\delta^{-k} \cdot \pi(k) ; \pi_{1}\left(k^{\prime}\right) ; \delta^{k} \cdot \phi(-\theta) ;
$$

donc la fonction $P$ reste la même. En mettant $\theta+\beta$ au lieu de $\theta$ la fonction $P$ reste encore invariable. Donc en vertu du $I^{\text {er }}$ théorème on aura

$$
\pi(k) \cdot \pi_{1}\left(k^{\prime}\right) \cdot \phi(-\theta)=u+u^{\prime} \cdot f(2 n+\mathrm{I}) \theta \cdot F(2 n+\mathrm{I}) \theta
$$

où $u$ et $u^{\prime}$ sont des fonctions entières de $\varphi(2 n+1) \theta$, la première du degré 3 et la seconde du degré $\mathrm{I}$. En mettant $\omega-\theta$ au lieu de $\theta$ les trois fonctions $\pi(k) ; \pi_{1}\left(k^{\prime}\right) ; \psi(-\theta)$ se changeront en

$$
-\pi^{\prime}(k) ;-\pi_{1}^{\prime}\left(k^{\prime}\right) ;-\phi \theta
$$


done

$$
\pi^{\prime}(k) \cdot \pi_{1}^{\prime}\left(k^{\prime}\right) \cdot \phi \theta=-u+u^{\prime} \cdot f(2 n+1) \theta \cdot F(2 n+1) \theta
$$

par suite

$$
\begin{gathered}
2 u=\pi(k) \cdot \pi_{1}\left(k^{\prime}\right) \cdot \psi(-\theta)-\pi^{\prime}(k) \cdot \pi_{1}^{\prime}\left(k^{\prime}\right) \cdot \phi \theta \\
2 u^{\prime} \cdot f(2 n+1) \theta \cdot F(2 n+1) \theta=\pi(k) \cdot \pi_{1}\left(k^{\prime}\right) \cdot \psi(-\theta)+\pi^{\prime}(k) \cdot \pi_{1}^{\prime}\left(k^{\prime}\right) \cdot \psi \theta .
\end{gathered}
$$

En changeant ici $\theta$ en $-\theta$ on voit que $u$ change de signe et que $u^{\prime}$ reste invariable; on aura donc

$$
u=a_{0} \varphi(2 n+\mathrm{I}) \theta+a_{1}(\varphi(2 n+\mathrm{I}) \theta)^{3} ; \quad u^{\prime}=b,
$$

où $a_{0}, a_{1}, b$ sont des quantités constantes par rapport à $\theta$. Il viendra donc:

Soit

$$
\begin{gathered}
\pi(k) \cdot \pi_{1}\left(k^{\prime}\right) \cdot \phi(-\theta)=a_{0} \varphi(2 n+1) \theta+a_{2}\{\varphi(2 n+1) \theta\}^{3} \\
+b \cdot f(2 n+1) \theta \cdot F(2 n+1) \theta
\end{gathered}
$$

$$
c_{k, k^{\prime}}=\frac{\mathrm{I}}{2 n+\mathrm{I}} \cdot \sum_{0}^{2 n} \sum_{0}^{2 n} \delta^{m k+\mu k^{\prime}} \cdot \varphi(m \alpha+\mu \beta)
$$

on aura en vertu de la formule $\left(3^{8}\right)$

$$
\phi \theta \cdot \phi(-\theta)=(2 n+\mathrm{I})^{2}\left\{c_{k, k^{\prime}}^{2}-\{\varphi(2 n+\mathrm{I}) \theta\}^{2}\right\} .
$$

L'équation (48) donnera donc en multipliant par $\phi \theta$

$$
\phi \theta=\frac{(2 n+1)^{2}\left\{c_{k, k^{\prime}}^{2}-\{\varphi(2 n+1) \theta\}^{2}\right\}}{a_{0} \varphi(2 n+\mathrm{I}) \theta+a_{1}\{\varphi(2 n+\mathrm{I}) \theta\}^{3}+b \cdot f(2 n+\mathrm{I}) \theta \cdot F(2 n+I) \theta} \cdot \pi(k) \cdot \pi_{1}\left(k^{\prime}\right) .
$$

Cette formule donne la fonction $\psi \theta$ à l'aide du produit des deux fonctions $\pi(k) ; \pi_{1}\left(k^{\prime}\right)$ si l'on connait seulement les trois constantes $a_{0}, a_{1}, b$. Or ces quantités se trouvent aisément comme il suit.

Faisons d'abord dans (48) $\varphi \theta=\frac{\mathrm{I}}{\mathrm{o}}$ après avoir divisé par $(\varphi \theta)^{3}$, on obtiendra: $a_{1}=-(2 n+1)^{3}$. Soit ensuite $\theta=0$ on aura en faisant pour abréger

$$
\left\{\begin{array}{l}
c_{k}=\frac{1}{2 n+1} \cdot \sum_{0}^{2 n} \sum_{0}^{2 n} \delta^{m k} \cdot \varphi(m \alpha+\mu \beta), \\
e_{k}=\frac{1}{2 n+1} \cdot \sum_{0}^{2 n} \sum_{0}^{2 n} \delta^{\mu k} \cdot \varphi(m \alpha+\mu \beta),
\end{array}\right.
$$




$$
\begin{gathered}
\pi(k)=c_{k} ; \quad \pi_{1}\left(k^{\prime}\right)=e_{k^{\prime}} ; \quad \phi(-\theta)=c_{k, k^{\prime}} ; \quad \varphi(2 n+\mathbf{1}) \theta=0 \\
f(2 n+\mathbf{I}) \theta \cdot F(2 n+\mathbf{I}) \theta=\mathbf{I}
\end{gathered}
$$

donc:

$$
b=(2 n+\mathrm{I})^{3} \cdot c_{k} \cdot e_{k^{\prime}} \cdot c_{k, k^{\prime}} \cdot
$$

Il reste à trouver le coefficient $a_{0}$. Or en multipliant membre à membre les deux équations $\left(47,47^{\prime}\right)$ on aura:

$$
\begin{aligned}
& \pi(k) \cdot \pi^{\prime}(k) \cdot \pi_{1}\left(k^{\prime}\right) \cdot \pi_{1}^{\prime}\left(k^{\prime}\right) \cdot \phi \theta \cdot \phi(-\theta) \\
= & -u^{2}+u^{\prime 2} \cdot\left\{f(2 n+1) \theta \cdot F(2 n+1) \theta^{\prime 2}\right.
\end{aligned}
$$

maintenant en vertu de la formule (50) on a

$$
\begin{aligned}
& \pi(k) \cdot \pi^{\prime}(k)=(2 n+\mathrm{I})^{2}\left\{c_{k}^{2}-\{\varphi(2 n+\mathrm{I}) \theta\}^{2}\right\} \\
& \pi_{1}\left(k^{\prime}\right) \cdot \pi_{1}^{\prime}\left(k^{\prime}\right)=(2 n+\mathrm{I})^{2}\left\{c_{k^{\prime}}^{2}-\{\varphi(2 n+\mathrm{I}) \theta\}^{2}\right\}
\end{aligned}
$$

donc en faisant $\varphi(2 n+\mathrm{I}) \theta=y$

$$
\begin{aligned}
& \left(a_{0} y+a_{1} y^{3}\right)^{2}-b^{2} \cdot\left(1-c^{2} y^{2}\right)\left(\mathrm{I}+e^{2} y^{2}\right) \\
= & (2 n+\mathrm{I})^{6}\left\{y^{2}-c_{k}^{2}\right\}\left\{y^{2}-e_{k^{\prime}}^{2}\right\}\left\{y^{2}-c_{k, k^{3}}^{2}\right\}
\end{aligned}
$$

et comparant de part et d'autre les coefficients de $y^{4}$ on obtiendra:

$$
2 a_{0} a_{1}+b^{2} e^{2} c^{2}=-(2 n+1)^{6} \cdot\left\{c_{k}^{2}+e_{k^{2}}^{2}+c_{k, k^{\prime}}^{2}\right\}
$$

donc en remettant les valeurs de $a_{1}$ et $b_{0}$

$$
a_{\theta}=\frac{1}{2}(2 n+\mathrm{I})^{3} \cdot\left\{c_{k}^{2}+e_{k^{\prime}}^{2}+c_{k, k^{\prime}}^{2}+e^{2} c^{2} c_{k}^{2} \cdot e_{k^{\prime}}^{2} \cdot c_{k, k^{\prime}}^{2}\right\}
$$

En substituant les valeurs de $a_{0}, a_{1}, b$ l'expression (5I) de $\phi \theta$ deviendra:

$$
\phi \theta=\frac{1}{2 n+1} \cdot \pi(k) \cdot \pi_{1}\left(k^{\prime}\right)
$$

$$
\times \frac{\{\varphi(2 n+1) \theta\}^{2}-c_{k, k^{\prime}}^{2}}{\{\varphi(2 n+\mathrm{I}) \theta\}^{3}-\frac{1}{2}\left\{c_{k}^{2}+e_{k^{\prime}}^{2}+c_{k, k^{\prime}}^{2}+e^{2} c^{2} c_{k}^{2} \cdot e_{k^{\prime}}^{2} \cdot c_{k, k^{\prime}}^{2}\right\} \cdot \varphi(2 n+1) \theta-c_{k} \cdot e_{k^{\prime}} \cdot c_{k, k^{\prime}} \cdot f(2 n+1) \theta \cdot F(2 n+1) \theta} .
$$

Il s'agit maintenant de trouver les fonetions $\pi(k)$ et $\pi_{1}\left(k^{\prime}\right)$. En désignant 
Recherches sur les fonctions elliptiques.

par $k$ et $k_{1}$ deux nombres entiers quelconques il est clair que les deux fonctions:

$$
\pi(k) \cdot \pi\left(k_{1}\right) \cdot \pi^{\prime}\left(k+k^{\prime}\right) \text { et } \pi_{1}(k) \cdot \pi_{1}\left(k_{1}\right) \cdot \pi_{1}^{\prime}\left(k+k_{1}\right)
$$

ne changeront pas de valeur en mettant $\theta+a$ et $\theta+\beta$ au lieu de $\theta$. Ces fonctions auront donc en vertu $d u i^{\text {er }}$ théorème la forme

$$
u+u^{\prime} f(2 u+1) \theta \cdot F(2 n+1) \theta
$$

où $u$ et $u^{\prime}$ sont des fonctions entières de $\varphi(2 n+1) \theta$. On démontrera aisément que $u^{\prime}$ sera constant et $u$ de la forme

Soit done:

$$
a_{0} \varphi(2 n+1) \theta+a_{1}\{\varphi(2 n+1) \theta\}^{3} .
$$

(54) $\left\{\begin{array}{c}\pi(k) \cdot \pi\left(k^{\prime}\right) \cdot \pi^{\prime}\left(k+k^{\prime}\right) \\ =a_{0} \varphi(2 n+1) \theta+a_{1}\{\varphi(2 n+1) \theta\}^{3}+b \cdot f(2 n+1) \theta \cdot F(2 n+1) \theta, \\ \pi_{1}(k) \cdot \pi_{1}\left(k^{\prime}\right) \cdot \pi_{1}^{\prime}\left(k+k^{\prime}\right) \\ =a_{0}^{\prime} \varphi(2 n+1) \theta+a_{1}^{\prime}\{\varphi(2 n+1) \theta\}^{3}+b^{\prime} \cdot f(2 n+1) \theta \cdot F(2 n+1) \theta .\end{array}\right.$

En traitant ces équations entièrement de la même manière que l'équation (48) on trouvera:

$$
\left\{\begin{array}{c}
a_{1}=-(2 n+\mathrm{I})^{3} ; \quad b=c_{k} \cdot c_{k^{\prime}} \cdot c_{k+k^{\prime}} \\
a_{0}=\frac{\mathrm{I}}{2}(2 n+\mathrm{I})^{3}\left\{c_{k}^{2}+c_{k^{\prime}}^{2}+c_{k+k^{\prime}}^{2}+e^{2} c^{2} c_{k}^{2} \cdot c_{k^{\prime}}^{2} \cdot c_{k+k^{\prime}}^{2}\right\} \\
a_{1}^{\prime}=-(2 n+\mathrm{I})^{3} ; \quad b^{\prime}=(2 n+\mathrm{I})^{3} \cdot e_{k} \cdot e_{k^{\prime}} \cdot e_{k+k^{\prime}} \\
a_{0}^{\prime}=\frac{1}{2}\left(2 n+i^{3} e_{k}^{2}+e_{k^{\prime}}^{2}+e_{k+k^{\prime}}^{2}+e^{2} c^{2} e_{k}^{2} \cdot e_{k^{\prime}}^{2} \cdot e_{k+k^{\prime}}^{2}\right\}
\end{array}\right.
$$

Cela posé, on obtiendra en multipliant les équations respectivement par $\pi\left(k+k^{\prime}\right)$ et $\pi_{1}\left(k+k^{\prime}\right)$ et remarquant que

$$
\begin{aligned}
& \pi\left(k+k^{\prime}\right) \cdot \pi^{\prime}\left(k+k^{\prime}\right)=(2 n+1)^{2}\left\{c_{k+k^{\prime}}^{2}-\varphi^{2}(n+1) \theta\right\} \\
& \pi_{1}\left(k+k^{\prime}\right) \cdot \pi_{1}^{\prime}\left(k+k^{\prime}\right)=(2 n+1)^{2}\left\{e_{k+k^{\prime}}^{2}-\varphi^{2}(n+1) \theta\right\}
\end{aligned}
$$


N. H. Abel.

$$
\left\{\begin{array}{c}
\pi\left(k+k^{\prime}\right)=\frac{1}{2 n+\mathrm{I}} \cdot \pi(k) \cdot \pi\left(k^{\prime}\right) \\
\times \frac{\{\varphi(2 n+\mathrm{I}) \theta\}^{2}-c_{k+k^{\prime}}^{2}}{\{\varphi(2 n+1) \theta\}^{3}-\frac{1}{2}\left(c_{k}^{2}+c_{k^{\prime}}^{2}+c_{k+k^{\prime}}^{2}+e^{2} c^{2} c_{k}^{2} \cdot c_{k^{\prime}}^{2} \cdot c_{k+k^{\prime}}^{2}\right) \varphi(2 n+\mathrm{I}) \theta-c_{k} \cdot c_{k^{\prime}} \cdot c_{k+k^{\prime}} \cdot f(2 n+\mathrm{I}) \theta \cdot F(2 n+1) \theta} \\
\pi_{1}\left(k+k^{\prime}\right)=\frac{1}{2 n+1} \cdot \pi_{1}(k) \cdot \pi_{1}\left(k^{\prime}\right) \\
\times \frac{\{\varphi(2 n+1) \theta\}^{2}-e_{k+k^{\prime}}^{2}}{\{\varphi(2 n+\mathrm{I}) \theta\}^{3}-\frac{1}{2}\left(e_{k}^{2}+e_{k^{\prime}}^{8}+e_{k+k^{\prime}}^{2}+e^{2} c^{2} e_{k}^{2} \cdot e_{k^{\prime}}^{2} \cdot e_{k+k^{\prime}}^{2}\right) \varphi(2 n+1) \theta-e_{k} \cdot e_{k^{\prime}} \cdot e_{k+k^{\prime}} \cdot f(2 n+\mathrm{I}) \theta \cdot F(2 n+1) \theta}
\end{array},\right.
$$

Ces formules donnent $\pi\left(k+k^{\prime}\right)$ en $\pi(k)$ et $\pi\left(k^{\prime}\right)$ et $\pi_{1}\left(k+k^{\prime}\right)$ en $\pi_{1}(k)$ et $\pi_{1}\left(k^{\prime}\right)$. Il est facile d'en tirer la valeur de $\pi(k)$ et $\pi_{1}(k)$. Soit $k^{\prime}=\mathrm{I}$ et mettons $k-1$ au lieu de $k$ nous aurons

$$
\left\{\begin{array}{l}
\pi(k)=\frac{\mathrm{I}}{2 n+1} \cdot \pi(\mathrm{I}) \cdot \pi(k-1) \cdot \frac{v_{k}}{t_{k}}, \\
\pi_{1}(k)=\frac{\mathrm{I}}{2 n+\mathrm{I}} \cdot \pi_{1}(\mathrm{I}) \cdot \pi_{1}(k-\mathrm{I}) \cdot \frac{v_{k}^{\prime}}{t_{k}^{\prime}}
\end{array}\right.
$$

où l'on a fait pour abréger

$$
\begin{gathered}
v_{k}=\{\varphi(2 n+\mathrm{I}) \theta\}^{2}-c_{k}^{2} ; \quad v_{k}^{\prime}=\{\varphi(2 n+\mathrm{I}) \theta\}^{2}-e_{k}^{2} \\
\left\{\begin{array}{r}
t_{k}^{\prime}=\{\varphi(2 n+\mathrm{I}) \theta\}^{3}-\frac{\mathrm{I}}{2}\left(e_{1}^{2}+e_{k-1}^{2}+e_{k}^{2}+e^{2} c^{2} e_{1}^{2} e_{k-1}^{2} e_{k}^{2}\right) \varphi(2 n+\mathrm{I}) \theta \\
-e_{1} e_{k-1} e_{k} \cdot f(2 n+\mathrm{I}) \theta \cdot F(2 n+\mathrm{I}) \theta \\
t_{k}=\{\varphi(2 n+\mathrm{I}) \theta\}^{3}-\frac{\mathrm{I}}{2}\left(c_{1}^{2}+c_{k-1}^{2}+c_{k}^{2}+e^{2} c^{2} c_{1}^{2} c_{k-1}^{2} c_{k}^{2}\right) \varphi(2 n+\mathrm{I}) \theta \\
-c_{1} c_{k-1} c_{k} \cdot f(2 n+\mathrm{I}) \theta \cdot F(2 n+\mathrm{I}) \theta
\end{array}\right.
\end{gathered}
$$

Cela posé les équations (57) donneront sur le champ en faisant $k=2,3, \ldots$ et éliminant ensuite:

$$
\left\{\begin{array}{l}
\pi(k)=\frac{\mathrm{I}}{(2 n+\mathrm{I})^{k-1}} \cdot\{\pi(\mathrm{I})\}^{k} \cdot \frac{v_{2} \cdot v_{3} \ldots v_{k}}{t_{2} \cdot t_{3} \ldots t_{k}} \\
\pi_{1}(k)=\frac{\mathrm{I}}{(2 n+\mathrm{I})^{k-1}} \cdot\left\{\pi_{1}(\mathrm{I})\right\}^{k} \cdot \frac{v_{\mathrm{s}}^{\prime} \cdot v_{\mathrm{s}}^{\prime} \ldots v_{k}^{\prime}}{t_{2}^{\prime} \cdot t_{\mathrm{s}}^{\prime} \ldots t_{k}^{\prime}}
\end{array}\right.
$$


De cette manière il reste seulement les deux fonctions $\pi(\mathrm{I})$ et $\pi_{1}(\mathrm{I})$, qui sont encore inconnues. Or si l'on fait $k=2 n+\mathrm{I}$ on a

$$
\begin{aligned}
& c_{k}=e_{k}=0 ; \quad \pi(2 n+\mathrm{I})=\pi(\mathrm{o})=(2 n+\mathrm{I})^{2} \cdot \varphi(2 n+\mathrm{I}) \theta=\pi_{1}(2 n+\mathrm{I}) \\
& v_{2 n+1}=\{\varphi(2 n+\mathrm{I}) \theta\}^{2} ; \quad t_{2 n+1}=\{\varphi(2 n+\mathrm{I}) \theta\}^{3}-c_{2 n}^{2}=v_{2 n} ; \quad t_{2 n+1}^{\prime}=v_{2 n}^{\prime}
\end{aligned}
$$

donc en substituant

$$
\begin{aligned}
& \{\pi(\mathrm{I})\}^{2 n+1}=(2 n+\mathrm{I})^{2 n+1} \cdot \frac{t_{2} \cdot t_{3} \ldots t_{2 n}}{v_{2} \cdot v_{3} \ldots v_{2 n-1}} \\
& \left\{\pi_{1}(\mathrm{I})\right\}^{2 n+1}=(2 n+\mathrm{I})^{2 n+1} \cdot \frac{t_{2}^{\prime} \cdot t_{3}^{\prime} \ldots t_{2 n}^{\prime}}{v_{2}^{\prime} \cdot v_{3}^{\prime} \ldots v_{2 n-1}^{\prime}}
\end{aligned}
$$

et de là

$$
\left\{\begin{array}{l}
\pi(\mathrm{I})=(2 n+\mathrm{I}) \cdot \sqrt[2 n+1]{\frac{t_{2} \cdot t_{3} \ldots t_{2 n}}{v_{2} \cdot v_{3} \ldots v_{2 n-1}}} \\
\pi_{1}(\mathrm{I})=(2 n+\mathrm{I}) \cdot \sqrt[2 n+1]{\frac{t_{2}^{\prime} \cdot t_{3}^{\prime} \ldots t_{2 n}^{\prime}}{v_{2}^{\prime} \cdot v_{3}^{\prime} \ldots v_{2 n-1}^{\prime}}}
\end{array}\right.
$$

Connaissant ainsi $\pi(\mathrm{I})$ et $\pi_{1}(\mathrm{I})$ on aura $\pi(k)$ et $\pi\left(k^{\prime}\right)$ par les équations (60) et ensuite $\phi \theta$ par l'équation (53).

On peut simplifier un peu les expressions de $\pi(\mathrm{I})$ et $\pi_{1}(\mathrm{I})$ en remarquant que $t_{2 n}=t_{2}, t_{2 n-1}=t_{3}, \ldots, v_{2 n-1}=v_{2}, v_{2 n-2}=v_{3}, \ldots$ etc. On obtiendra ainsi

$$
\left\{\begin{array}{l}
\pi(\mathrm{I})=(2 n+\mathrm{I}) \cdot \sqrt[2 n+1]{\left(\frac{t_{2} \cdot t_{3} \ldots t_{n}}{v_{2} \cdot v_{3} \ldots v_{n}}\right)^{2} \cdot t_{n+1}}, \\
\pi_{1}(\mathrm{I})=(2 n+1) \cdot \sqrt[2 n+1]{\left(\frac{t_{2}^{\prime} \cdot t_{3}^{\prime} \ldots t_{n}^{\prime}}{v_{2}^{\prime} \cdot v_{3}^{\prime} \ldots v_{n}^{\prime}}\right)^{2} \cdot t_{n+1}^{\prime}} .
\end{array}\right.
$$

Les quantités sous les radicaux sont représentées sous une forme fractionnaire mais selon ce qui précède elles sont réellement des fonctions entières. On pourra trouver ces fonctions par un procédé particulier que nous allons exposer.

Multiplions l'expression de $\pi(k)$ par $\pi^{\prime}(k)$; nous obtiendrons, en remarquant que $\pi(k) \cdot \pi^{\prime}(k)=(2 n+1)^{2} \cdot\left\{c_{k}^{2}-\{\varphi(2 n+1) \theta\}^{2}\right\}=-(2 n+1)^{2} v_{k}$,

$$
\pi^{\prime}(k) \cdot\{\pi(\mathrm{I})\}^{k}=-(2 n+\mathrm{I})^{k+1} \cdot \frac{t_{2} \cdot t_{3} \ldots t_{k}}{v_{3} \cdot v_{3} \ldots v_{k-1}} .
$$


Cela posé, soit

$$
\frac{t_{2} \cdot t_{3} \ldots t_{k}}{v_{2} \cdot v_{3} \ldots v_{k-1}}=S_{k}
$$

nous aurons

$$
v_{k} . S_{k+1}=t_{k+1} . S_{k} ; \quad v_{k-1} . S_{k}=t_{k} \cdot S_{k-1} .
$$

De là on tire en multipliant la $\mathrm{I}^{\text {ere }}$ équation par $c_{k-1}$ et la seconde par $-c_{k+1} \cdot \frac{\theta_{k}}{v_{k-1}}$ et ensuite ajoutant:

$$
\mathrm{O}=c_{k-1} \cdot v_{k} \cdot S_{k+1}-\left(c_{k-1} t_{k+1}+c_{k+1} \cdot \theta_{k}\right) \cdot S_{k}+c_{k+1} \cdot \frac{t_{k} \cdot \theta_{k}}{v_{k-1}} \cdot S_{k-1} .
$$

Cela posé si l'on désigne par $\theta_{k}$ la valeur de $t_{k}$ en y changeant le signe de $f(2 n+\mathrm{I}) \boldsymbol{\theta} \cdot \boldsymbol{F}(2 n+\mathbf{1}) \boldsymbol{\theta}$, les coefficients de $S_{k}$ et $S_{k-1}$ seront divisibles par $v_{k}$. En effet on a d'abord

$$
t_{k} . \theta_{k}=\left\{\varphi^{2}(2 n+1) \theta-c_{1}^{2}\right\}\left\{\varphi^{2}(2 n+1) \theta-c_{k}^{2}\right\}\left\{\varphi^{2}(2 n+1) \theta-c_{k-1}^{2}\right\},
$$

c'est à dire

$$
t_{k} \cdot \theta_{k}=v_{1} \cdot v_{k} \cdot v_{k-1} .
$$

On troave encore à l'aide de la formule (59)

$$
\begin{aligned}
c_{k-1} t_{k+1}+c_{k+1} \cdot \theta_{k} & =\left(c_{k-1}+c_{k+1}\right) \cdot \varphi(2 n+1) \theta \cdot\left\{\varphi^{2}(2 n+1) \theta-c_{k}^{2}\right\} \\
& =\left(c_{k-1}+c_{k+1}\right) \cdot \varphi(2 n+1) \theta \cdot v_{k} .
\end{aligned}
$$

On aura donc en substituant dans l'équation (65) et divisant ensuite par $v_{k}$ :

$$
\begin{gathered}
c_{k-1} \cdot S_{k+1}-\left(c_{k-1}+c_{k+1}\right) \cdot \varphi(2 n+1) \theta \cdot S_{k} \\
+c_{k+1} \cdot\left\{\varphi^{2}(2 n+1) \theta-c_{1}^{2}\right\} \cdot S_{k-1}=0 .
\end{gathered}
$$

Cette formule donne une loi très simple pour former successivement les fonctions $S_{k}$ en connaissant les deux $S_{1}$ et $S_{2}$. Or on a

$$
S_{1}=-\frac{\pi^{\prime}(r) \cdot \pi(1)}{(2 n+1)^{2}}=v_{1}=\varphi^{2}(2 n+1) \theta-c_{1}^{2} \text { et } S_{2}=t_{2} .
$$

Par là on voit donc que les quantités $S_{z}, S_{4}, \ldots, S_{k}$ déterminées par la 
formule (66) en faisant $k=2,3, \ldots$ seront des fonctions entières. En faisant $k=2 n$ on aura la valeur de

et

$$
S_{2 n}=\frac{t_{2} \cdot t_{3} \ldots t_{2 n}}{v_{2} \cdot v_{3} \ldots v_{2 n-1}}
$$

$$
\pi(\mathrm{I})=(2 n+\mathrm{I}) \cdot \sqrt[2 n+1]{S_{2 n}} .
$$

On aura en même temps en vertu de la formule (6o)

$$
\pi(k)=\frac{\mathrm{I}}{(2 n+\mathbf{I})^{k-1}} \cdot\{\pi(\mathbf{I})\}^{k} \cdot \frac{v_{k}}{S_{k}}
$$

En faisant

$$
S_{k}^{\prime}=\frac{t_{2}^{\prime} \cdot t_{3}^{\prime} \ldots t_{k}^{\prime}}{v_{2}^{\prime} \cdot v_{3}^{\prime} \ldots v_{k-1}^{\prime}}
$$

on aura entièrement de la même manière

$$
\begin{gathered}
e_{k-1} \cdot S_{k+1}^{\prime}-\left(e_{k-1}+e_{k+1}\right) \cdot \varphi(2 n+1) \theta \cdot S_{k}^{\prime} \\
+e_{k+1} \cdot\left\{\varphi^{2}(2 n+1) \theta-e_{1}^{2}\right\} \cdot S_{k-1}^{\prime}=0 .
\end{gathered}
$$

$\S 3$.

Théorème IV. Soient $a_{0}, a_{1}, a_{2}, \ldots, b_{0}, b_{1}, b_{2}, \ldots$ des quantités en nombre quelconque et dont l'une au moins soit variable. Si l'on désigne les racines de l'équation

$$
\begin{gathered}
\left(a_{0}+a_{1} x+a_{2} x^{2}+\ldots+a_{m} x^{m}\right)^{2} \\
-\left(b_{0}+b_{1} x+b_{2} x^{2}+\ldots+b_{n} x^{n}\right)^{2}\left(1-c^{2} x^{2}\right)\left(1+e^{2} x^{2}\right)=0
\end{gathered}
$$

par

$$
\varphi \theta_{1}, \varphi \theta_{2}, \varphi \theta_{3}, \ldots, \varphi \theta_{\mu}
$$

je dis qu'on aura

$$
\varphi\left( \pm \theta_{1} \pm \theta_{2} \pm \theta_{3} \pm \ldots \pm \theta_{\mu}\right),=\text { à une constante }
$$

en déterminant convenablement le signe des quantités $\theta_{1}, \theta_{2}, \ldots, \theta_{\mu}$. 
Démonstration. Soient pour abréger

$$
\left\{\begin{array}{l}
p=a_{0}+a_{1} x+\ldots+a_{m} x^{m}, \\
q=b_{0}+b_{1} x+\ldots+b_{n} x^{n}
\end{array}\right.
$$

et faisons

$$
\phi(x)=p^{2}-q^{2}\left(\mathrm{I}-c^{2} x^{2}\right)\left(\mathrm{I}+e^{2} x^{2}\right) .
$$

En mettant pour $x$ l'une quelconque des quantités $\varphi \theta_{1}, \varphi \theta_{2}, \ldots$ on aura (75) $\phi(\varphi \theta)=p^{2}-q^{2} \cdot\left(\mathrm{I}-c^{2} \varphi \theta\right) \cdot\left(\mathrm{I}+e^{2} \varphi^{2} \theta\right)=\mathrm{O}=p^{2}-q^{2}(f \theta)^{2} \cdot(F \theta)^{2}$.

En différentiant cette équation par rapport à $\theta$ et $a_{0}, a_{1}, \ldots, b_{0}, b_{1}, \ldots$ il viendra

$$
\psi^{\prime}(\varphi \theta) \cdot f \theta \cdot F \theta \cdot d \theta+2 p \grave{o p}-2 q \grave{o} q(f \theta)^{2} \cdot(F \theta)^{2}=0
$$

où le signe de différentiation $\delta$ se rapporte aux seules quantités $a_{0}, a_{1}, \ldots$, $b_{0}, \ldots$ Maintenant la même équation (75) donne

$$
p=s q f \theta . F \theta \text { où } \varepsilon= \pm \mathrm{I} .
$$

En vertu de cette équation on aura

$$
2 p \delta p-2 q \delta q(f \theta)^{2}(F \theta)^{2}=2 \varepsilon f \theta . F \theta\{q \delta p-p \delta q\} .
$$

L'équation (76) deviendra donc en substituant et divisant par $f \theta . F \theta$ :

d'où l'on tire

$$
\phi^{\prime}(\varphi \theta) \cdot d \theta+2 \varepsilon(q \dot{\delta} p-p \dot{\partial} q)=0
$$

$$
\varepsilon d \theta=\frac{2(p \delta q-q \delta p)}{\phi^{\prime}(\varphi \theta)}
$$

La quantité $2(p \grave{o} q-q \grave{o} p)$ est une fonction entière de $x=\varphi \theta$ que nous désignerons par $\lambda(\varphi \theta)$. On aura par suite en mettant pour $\theta$ ces valeurs $\theta_{1}, \theta_{2}, \theta_{3}, \ldots, \theta_{\mu}$ et désignant les valeurs correspondantes de $\varepsilon$ par $\varepsilon_{1}, \varepsilon_{2}$, $\varepsilon_{3}, \ldots, \varepsilon_{\mu}$ les équations suivantes:

$$
\varepsilon_{1} d \theta_{1}=\frac{\lambda\left(\varphi \theta_{1}\right)}{\zeta^{\prime}\left(\varphi \theta_{1}\right)} ; \quad \varepsilon_{2} \lambda \theta_{2}=\frac{\lambda\left(\varphi \theta_{2}\right)}{\zeta^{\prime}\left(\varphi \theta_{2}\right)} ; \quad \ldots \quad ; \quad \varepsilon_{\mu} d \theta_{\mu}=\frac{\lambda\left(\varphi \theta_{\mu}\right)}{\phi^{\prime}\left(\varphi \theta_{\mu}\right)}
$$


qui ajoutées membres à membres donneront celle-ci

$$
\varepsilon_{1} d \theta_{1}+\varepsilon_{2} d \theta_{2}+\ldots+\varepsilon_{\mu} d \theta_{\mu}=\frac{\lambda\left(\varphi \theta_{1}\right)}{\psi^{\prime}\left(\varphi \theta_{1}\right)}+\frac{\lambda\left(\varphi \theta_{2}\right)}{\psi^{\prime}\left(\varphi \theta_{2}\right)}+\ldots+\frac{\lambda\left(\varphi \theta_{u}\right)}{\psi^{\prime}\left(\varphi \theta_{\mu}\right)} .
$$

Cela posé il est facile de voir que le degré de la fonction entière $\lambda(x)$ est moindre que celui de la fonction $\psi^{\prime}(x)$; donc en vertu d'une formule connue le second membre de l'équation précédente s'évanouira. Il viendra donc

$$
\varepsilon_{1} d \theta_{1}+\varepsilon_{2} d \theta_{2}+\varepsilon_{3} d \theta_{3}+\ldots+\varepsilon_{\mu} d \theta_{\mu}=0
$$

d'où l'on tire en intégrant:

$$
\varepsilon_{1} \theta_{1}+\varepsilon_{2} \theta_{2}+\varepsilon_{3} \theta_{3}+\ldots+\varepsilon_{\mu} \theta_{\mu}=\text { à une constante }
$$

et de là

$$
\varphi\left(\varepsilon_{1} \theta_{1}+\varepsilon_{2} \theta_{2}+\varepsilon_{3} \theta_{3}+\ldots+\varepsilon_{\mu} \theta_{\mu}\right)=C .
$$

Le théorème est donc démontré.

La démonstration précédente suppose que toutes les racines de l'équation soient inégales, mais il est évident que la formule (83) aura encore lieu si quelques-unes des quantités $\varphi \theta_{1}, \varphi \theta_{2}, \varphi \theta_{3}, \ldots, \varphi \theta_{\mu}$ deviendront égales entre elles.

La valeur de $\varepsilon_{1}, \varepsilon_{2}, \ldots, \S_{\mu}$ n'est pas arbitraire; elle est déterminée par l'équation $p=\varepsilon q f \theta . F \theta$, c'est-à-dire

$$
\begin{gathered}
a_{0}+a_{1} \varphi \theta_{k}+a_{2}\left(\varphi \theta_{k}\right)^{2}+\ldots+a_{m}\left(\varphi \theta_{k}\right)^{m} \\
=\varepsilon_{k} \cdot\left(b_{0}+b_{1} \varphi \theta_{k}+b_{2}\left(\varphi \theta_{k}\right)^{2}+\ldots+b_{n}\left(\varphi \theta_{k}\right)^{n}\right) \cdot f \theta_{k} \cdot F \theta_{k} .
\end{gathered}
$$

Les quantités $\theta_{1}, \theta_{2}, \ldots, \theta_{2}$ sont en vertu de l'équation $\psi(x)=0$ des fonctions de $a_{0}, a_{1}, \ldots, b_{0}, b_{1}, \ldots$. Si un certain nombre $k$ de ces dernières quantités sont indéterminées on pourra en général les déterminer de la manière que $k$ des quantités $\theta_{1}, \theta_{2}, \ldots, \theta_{n}$ soient données. Soient done $\theta_{1}, \theta_{2}, \ldots, \theta_{k}$ données, les quantités $\theta_{k+1}, \theta_{k+2}, \ldots, \theta_{\mu}$ deviendront des fonctions de celles-là. Le cas le plus simple et le plus important est celui où $\mu-k$ a la valeur la plus petite possible. Or il est clair qu'on peut disposer de $\mu-1$ des quantités $a_{0}, a_{1}, \ldots, b_{0}, b_{1}, \ldots$; donc on peut regarder les $\mu$ - I quantités $\theta_{1}, \theta_{2}, \ldots, \theta_{\mu-1}$ comme données et en- 
suite déterminer $\varphi \theta_{\mu}$ en fonction de $\varphi \theta_{1}, \varphi \theta_{2}, \ldots, \varphi \theta_{n-1}$ de la sorte que l'équation (83) soit satisfaite. Soit

$$
\varphi \theta_{\mu}=\phi\left\{\varphi \theta_{1}, \varphi \theta_{2}, \ldots, \varphi \theta_{\mu-1}\right\}
$$

on tire de l'équation (82) une autre valeur de $\varphi \theta_{\mu}$ savoir

donc

$$
\varphi \theta_{\mu}=-\varepsilon_{\mu} \varphi\left(\varepsilon_{1} \theta_{1}+\varepsilon_{2} \theta_{2}+\ldots+\varepsilon_{\mu-1} \theta_{\mu-1}-C^{\prime}\right)
$$

$$
\varphi\left(\varepsilon_{1} \theta_{1}+\varepsilon_{2} \theta_{2}+\ldots+\varepsilon_{\mu-1} \theta_{\mu-1}-C\right)=-\varepsilon_{\mu} \cdot \psi\left\{\varphi \theta_{1}, \varphi \theta_{2}, \ldots, \varphi \theta_{\mu-1}\right\} .
$$

Cette formule dans laquelle toutes les quantités $\theta_{1}, \theta_{2}, \ldots, \theta_{i,-1}$, aussi bien que $\varepsilon_{1}, \varepsilon_{2}, \ldots, \varepsilon_{k-1}$, sont arbitraires, exprime la propriété fondamentale des fonctions elliptiques. En posant $\varepsilon_{1}=\varepsilon_{2}=\ldots=\varepsilon_{\mu-1}$, on a

$$
\varphi\left(\theta_{1}+\theta_{2}+\theta_{3}+\ldots+\theta_{\mu-1}-C\right)=-\varepsilon_{\mu} \psi\left(\varphi \theta_{1}, \varphi \theta_{2}, \ldots, \varphi \theta_{\mu-1}\right) .
$$

Cela posé, considérons quelques cas particulicrs.

I. Soient

$$
\left\{\begin{array}{l}
p=a_{0} x+a_{1} x^{3}+\ldots+a_{n} x^{2 n+1}=\lambda(x), \\
q=b_{0}+b_{1} x^{2}+\ldots+b_{n-1} x^{2 n-2}=\lambda_{1}(x)
\end{array}\right.
$$

dans ce cas l'équation (7 I

$$
p^{2}-q^{2}\left(\mathrm{I}-c^{2} x^{2}\right)\left(\mathrm{I}+e^{2} x^{2}\right)=\mathrm{O}
$$

sera du degré $4 n+2$, et ses racines seront deux à deux égales mais de signes contraires. Soient donc

$$
\theta_{2 n+2}=-\theta_{1} ; \quad \theta_{2 n+3}=-\theta_{2} ; \ldots ; \theta_{4 n+2}=-\theta_{2 n+1} .
$$

Supposons $\theta_{1}, \theta_{2}, \ldots, \theta_{2 n}$ donnés et $\varepsilon_{1}=\varepsilon_{2}=\ldots=\varepsilon_{2 n}=1$, l'équation (77) donnera celles-ci

$$
\begin{gathered}
\lambda\left(\varphi \theta_{1}\right)=\lambda_{1}\left(\varphi \theta_{1}\right) \cdot f \theta_{1} \cdot F \theta_{1} ; \quad \lambda\left(\varphi \theta_{2}\right)=\lambda_{1}\left(\varphi \theta_{2}\right) \cdot f \theta_{2} \cdot F \theta_{2} ; \ldots ; \\
\lambda\left(\varphi \theta_{2 n}\right)=\lambda_{1}\left(\varphi \theta_{2 n}\right) \cdot f \theta_{2 n} . F \theta_{2 n} .
\end{gathered}
$$

$\varepsilon_{2 n+1}, \Xi_{2 n+2}, \ldots, \varepsilon_{4 n+2}$ sont déterminés par les équations:

$$
\lambda\left(\varphi \theta_{2 n+1+m}\right)=\varepsilon_{2 n+1+m} \lambda_{1}\left(\varphi \theta_{2 n+1+m}\right) \cdot f \theta_{2 n+1+m} . F \theta_{2 n+1+m},
$$


c'est-à-dire en remarquant que $\theta_{2 n+1+m}=-\theta_{m}$

$$
-\lambda\left(\varphi \theta_{m}\right)=\varepsilon_{2 n+1+m} \lambda_{1}\left(\varphi \theta_{m}\right) \cdot f \theta_{m} \cdot F \theta_{m}
$$

donc en vertu des équations (90)

$$
\varepsilon_{2 n+1+n}=-1 ; \text { si } m>0 \text { et } m<2 n+1 \text {. }
$$

On aura de même

$$
\varepsilon_{4 n+2}=-\varepsilon_{2 n+1} .
$$

La formule (82) deviendra donc

$$
2 \theta_{1}+2 \theta_{2}+\ldots+2 \theta_{2 n}+2 \varepsilon_{2 n+1} \theta_{2 n+1}=C
$$

d'où

$$
\varphi\left(\theta_{1}+\theta_{2}+\theta_{3}+\ldots+\theta_{2 n}+a\right)=-\varepsilon \cdot \varphi \theta_{2 n+1} .
$$

$\varepsilon$ est égal à $\pm \mathrm{I}$ et déterminé par l'équation

$$
\varepsilon=\frac{\lambda_{1}\left(\varphi \theta_{2 n+1}\right) \cdot f \theta_{2 n+1} \cdot F \theta_{2 n+1}}{\lambda\left(\varphi \theta_{2 n+1}\right)}
$$

$a$ est une quantité constante que nous allons déterminer.

L'équation (89) nous montre que $b_{0}^{2}$ est égal au produit des racines, donc

on en tire:

$$
b_{0}^{2}=\varphi^{2} \theta_{1} \cdot \varphi^{2} \theta_{2} \ldots \varphi^{2} \theta_{2 n} \cdot \varphi^{2} \theta_{2 n+1}
$$

$$
\varphi \theta_{2 n+1}= \pm \frac{b_{0}}{\varphi \theta_{1} \cdot \varphi \theta_{2} \ldots \varphi \theta_{2 n}}
$$

Ia quantité $b_{0}$ se détermine à l'aide des équations (9o). Comme elles sont linéaires par rapport aux quantités $a_{0}, a_{1}, \ldots, b_{0}, b_{1}, \ldots$, elles donneront $b_{0}$ et par suite $\varphi \theta_{2 n+1}$ en fonction rationnelle des quantités

$$
\varphi \theta_{1}, \varphi \theta_{2}, \ldots, \varphi \theta_{2 n} ; f \theta_{1} . F \theta_{1} ; f \theta_{2} . F \theta_{2} ; \ldots ; f \theta_{2 n} . F \theta_{2 n} .
$$

Pour déterminer la constante $a$ soient $\theta_{1}=\theta_{2}=\ldots=\theta_{2 n}=0$.

Alors il est clair par la forme de l'équation (89) qu'on doit avoir $b_{0}=a_{0}=b_{1}=a_{1}=\ldots=a_{n-1}=0$. Cette équation se réduit alors à

$$
x^{4 n+2}=0
$$


et par suite dans ce cas $\varphi \theta_{2 n+1}=0$, donc Ja formule (92) donnera

et par suite

$$
\varphi a=0
$$

$$
\varphi\left(\theta_{1}+\theta_{2}+\theta_{3}+\ldots+\theta_{2 n}\right)= \pm \frac{l_{0}}{\varphi \theta_{1} \cdot \varphi \theta_{2} \ldots \varphi \theta_{2 n}} .
$$

Supposons par ex. $n=1$, on aura

$$
\varphi\left(\theta_{1}+\theta_{2}\right)= \pm \frac{b_{0}}{\varphi \theta_{1} \cdot \varphi \theta_{2}}
$$

et pour déterminer $b_{0}$ on aura les deux équations

$$
\begin{aligned}
& a_{0} \varphi \theta_{1}+\varphi^{3} \theta_{1}=b_{0} . f \theta_{1} \cdot F \theta_{1}, \\
& a_{0} \varphi \theta_{2}+\varphi^{3} \theta_{2}=b_{0} . f \theta_{2} . F \theta_{2} .
\end{aligned}
$$

On en tire en éliminant $a_{0}$

$$
b_{0}=\frac{\varphi \theta_{1} \cdot \varphi \theta_{3} \cdot\left\{\varphi^{2} \theta_{1}-\varphi^{2} \theta_{1}\right\}}{\varphi \theta_{2} \cdot f \theta_{1} \cdot F \theta_{1}-\varphi \theta_{1} \cdot f \theta_{2} \cdot F \theta_{3}}
$$

donc en substituant dans la formule $\left(94^{\prime}\right)$

$$
\varphi\left(\theta_{1}+\theta_{2}\right)= \pm \frac{\varphi^{2} \theta_{1}-\varphi^{2} \theta_{2}}{\varphi \theta_{2} \cdot f \theta_{1} \cdot F \theta_{1}-\varphi \theta_{1} \cdot f \theta_{2} \cdot F \theta_{2}} .
$$

Pour déterminer le signe, soit $\theta_{2}=0$, on aura $\varphi \theta_{1}=\mp \varphi \theta_{1}$; donc il faut prendre le signe inférieur et par suite

$$
\varphi\left(\theta_{1}+\theta_{2}\right)=\frac{\varphi^{2} \theta_{1}-\varphi^{2} \theta_{2}}{\varphi \theta_{1} \cdot f \theta_{2} \cdot F \theta_{2}-\varphi \theta_{2} \cdot f \theta_{1} \cdot F \theta_{1}} .
$$

Si on multiplie haut et bas de la fraction par le dénominateur en changeant le signe $d u$ second terme, on trouve en réduisant

$$
\varphi\left(\theta_{1}+\theta_{2}\right)=\frac{\varphi \theta_{1} \cdot f \theta_{2} \cdot F \theta_{2}+\varphi \theta_{2} \cdot f \theta_{1} \cdot F \theta_{1}}{\mathrm{I}+e^{2} c^{2} \varphi^{2} \theta_{1} \cdot \varphi^{2} \theta_{1}}
$$

c'est-à-dire la $1^{\text {ire }}$ des formules (Io), tome II, pag. 105.

la formule $(9 \mathrm{I})$ donne

$$
\varphi\left(\theta_{1}+\theta_{2}+\ldots+\theta_{2 n}+\varepsilon_{2 n+1} \cdot \theta_{2 n+1}\right)=C .
$$

Si l'on pose $\theta_{1}=\theta_{2}=\ldots=\theta_{2 n}=0$, on aura comme nous avons vu 
plus haut $\theta_{2 n+1}$ et par suite $C=0$. En faisant en même temps $\varepsilon_{2 n+1}=\mathrm{I}$ il est clair qu'on aura en vertu de ce qui précède ce théorème:

Théorème V. Si l'expression

$$
\frac{a_{1} \varphi \theta+a_{1}(\varphi \theta)^{3}+\ldots+a_{n}(\varphi \theta)^{2 n+1}-\left(b_{0}+b_{1}(\varphi \theta)^{2}+\ldots+b_{n-1}(\varphi \theta)^{2 n-9}\right) \cdot f \theta \cdot F \theta}{\left(\varphi^{2} \theta-\varphi^{2} \theta_{1}\right)\left(\varphi^{2} \theta-\varphi^{2} \theta_{2}\right)\left(\varphi^{2} \theta-\varphi^{2} \theta_{3}\right) \ldots\left(\varphi^{2} \theta-\varphi^{2} \theta_{2 n}\right)\left(\varphi^{2} \theta-\varphi^{2} \theta_{3 n+1}\right)}
$$

reste finie en attribuant à la quantité $\theta$ les valeurs $\theta_{1}, \theta_{2}, \ldots, \theta_{2 n+1}$ on aura toujours

$$
\varphi\left(\theta_{1}+\theta_{2}+\theta_{3}+\ldots+\theta_{2 n}+\theta_{2 n+1}\right)=0 .
$$

En faisant $\theta_{1}=\theta_{2}=\ldots=\theta_{2 n+1}=\alpha$ on aura comme corollaire ce théorème:

Théorème VI. Si l'expression

$$
\frac{a_{0} \varphi \theta+a_{1}(\varphi \theta)^{3}+\ldots+a_{n}(\varphi \theta)^{2 n+1}-\left(b_{0}+b_{1}(\varphi \theta)^{2}+\ldots+b_{n-1}(\varphi \theta)^{2 n-2}\right) \cdot f \theta . F \theta}{\left(\varphi^{2} \theta-\varphi^{2} \alpha\right)^{2 n+1}}
$$

se réduit à une quantité finie en faisant $\theta=\alpha$ on aura

ou bien si la fonction

$$
\varphi(2 n+1) \alpha=0
$$

$a_{0} \varphi \theta+a_{1}(\varphi \theta)^{3}+\ldots+a_{n}(\varphi \theta)^{2 n+1}-\left(b_{0}+b_{1}(\varphi \theta)^{2}+\ldots+b_{n-1}(\varphi \theta)^{2 n-2}\right) \cdot f \theta \cdot F \theta$

et les dérivées par rapport à $\theta$ jusqu'à l'ordre $2 n$ incl. se réduisent à zéro pour $\theta=\alpha$ on aura

$$
\varphi(2 n+1) \alpha=0 .
$$

On peut encore énoncer ce dernier théorème comme il suit.

Théorème VII. Si les quantités $a_{0}, a_{1}, \ldots, b_{0}, b_{1}, \ldots$ sont telles que l'équation

$$
\begin{gathered}
\left(a_{0} x+a_{1} x^{3}+\ldots+a_{n} x^{2 n+1}\right)^{2} \\
-\left(b_{0}+l_{1} x^{2}+\ldots+b_{n-1} x^{2 n-2}\right)\left(\mathrm{I}-c^{2} x^{2}\right)\left(\mathrm{I}+e^{2} x^{2}\right)=a_{n}^{2}\left\{x^{2}-\varphi^{2} \alpha\right\}^{2 n+1}
\end{gathered}
$$

a lieu, la quantité $\alpha$ satisfera nécessairement à l'équation

$$
\varphi(2 n+\mathrm{I}) a=0
$$

c'est-à-dire on doit avoir $\alpha=\frac{m \omega+m^{\prime} \bar{\omega} i}{2 n+1}$ oì $m$ et $m^{\prime}$ sont des nombres entiers. 
2. Soient maintenant

$$
\left\{\begin{array}{l}
p=a_{0}+a_{1} x^{2}+\ldots+a_{n} x^{2 n}=\lambda(x), \\
q=b_{0} x+b_{1} x^{3}+\ldots+b_{n-2} x^{2 n-3}=\lambda_{1}(x) .
\end{array}\right.
$$

Dans ce cas l'équation

$$
p^{2}-q^{2}\left(1-c^{2} x^{2}\right)\left(1+e^{2} x^{2}\right)=0
$$

sera du degré $4 n$ et ses racines seront deux à deux égales mais de signe contraire. En faisant $\theta_{2 n+1}=-\theta_{1}, \theta_{2 n+2}=-\theta_{2}, \ldots, \theta_{4 n}=-\theta_{2 n}$, $\varepsilon_{1}=\varepsilon_{2}=\ldots=\varepsilon_{2 n-1}=\mathrm{I}$ on aura pour déterminer $a_{0}, a_{1}, \ldots, b_{0}, b_{1} \ldots$ les équations suivantes:

$$
\begin{gathered}
\lambda\left(\varphi \theta_{1}\right)=\lambda_{1}\left(\varphi \theta_{1}\right) \cdot f \theta_{1} . F \theta_{1} ; \quad \lambda\left(\varphi \theta_{2}\right)=\lambda_{1}\left(\varphi \theta_{2}\right) \cdot f \theta_{2} . F \theta_{2} ; \ldots ; \\
\lambda\left(\varphi \theta_{2 n-1}\right)=\lambda_{1}\left(\varphi \theta_{2 n-1}\right) \cdot f \theta_{2 n-1} . F \theta_{2 n-1} .
\end{gathered}
$$

On aura ensuite

$$
\begin{gathered}
\varepsilon_{2 n}=\frac{\lambda_{1}\left(\varphi \theta_{2 n}\right) \cdot f \theta_{2 n} \cdot F \theta_{2 n}}{\lambda\left(\varphi \theta_{2 n}\right)}, \\
\varepsilon_{2 n+1}=\varepsilon_{2 n+2}=\ldots=\varepsilon_{4 n-1}=-\mathrm{I}, \quad \varepsilon_{4 n}=-\varepsilon_{2 n} .
\end{gathered}
$$

La formule (82) donnera donc

$$
2 \theta_{1}+2 \theta_{2}+\ldots+2 \theta_{2 n-1}+2 \varepsilon_{2 n} \theta_{2 n}=\text { const., }
$$

de là

$$
\varphi\left(\theta_{1}+\theta_{2}+\ldots+\theta_{2 n-1}+a\right)=-\varepsilon_{2 n} \varphi \theta_{2 n} .
$$

En faisant $\theta_{1}=\theta_{2}=\ldots=\theta_{2 n-1}=0$ on aura

$$
\begin{aligned}
& b_{0}=b_{1}=\ldots=b_{n-2}=0, \\
& a_{0}=a_{1}=\ldots=a_{n-1}=0 ;
\end{aligned}
$$

donc l'équation $(96)$ deviendra $x^{4 n}=0$. Toutes les racines sont donc alors égales à zéro et par suite $\varphi \theta_{2 n}=0$. L'équation (98) donnera donc $\varphi a=0$ et par conséquent

$$
\varphi\left(\theta_{1}+\theta_{2}+\ldots+\theta_{2 n-1}\right)= \pm \varphi \theta_{2 n},
$$

$\varphi \theta_{2 n}$ se trouve en remarquant que $a_{0}^{2}$ est le produit de toutes les racines, donc

$$
a_{0}^{2}=\varphi^{2} \theta_{1} \cdot \varphi^{2} \theta_{2} \ldots \varphi^{2} \theta_{2 n}
$$


et de là

done enfin

$$
\varphi \theta_{2 n}= \pm \frac{a_{0}}{\varphi H_{1} \cdot \varphi \theta_{2} \ldots \varphi \theta_{2 n-1}}
$$

$$
\varphi\left(\theta_{1}+\theta_{2}+\ldots+\theta_{2 n-1}\right)= \pm \frac{a_{0}}{\varphi \theta_{1} \cdot \varphi \theta_{2} \ldots \theta_{2 n-1}} .
$$

$a_{0}$ et par suite le second membre de cette équation est une fonction rationnelle des quantités:

$$
\varphi \theta_{1}, \varphi \theta_{2} \ldots, \varphi \theta_{2 n-1} ; f \theta_{1} . F \theta_{1} ; f \theta_{2} . F \theta_{2} ; \ldots ; f \theta_{2 n-1}, F \theta_{2 n-1} .
$$

On pent remarquer que la formule (99) doit se déduire de la formule (94), en $y$ faisant $\theta_{2 n}=0$.

L'équation $\left(97^{\prime}\right)$ donne encore

$$
\varphi\left(\theta_{1}+\theta_{2}+\ldots+\theta_{2 n-1}+\varepsilon_{2 n} \theta_{2 n}\right)=C .
$$

On trouve $C^{\gamma}=0$. Si donc $\varepsilon_{2 n}=\mathrm{I}$ on aura le théorème suivant:

Théorème VIII. Si l'expression

$$
\frac{\left\{a_{0}+a_{1}(\varphi \theta)^{2}+a_{2}(\varphi \theta)^{4}+\ldots+a_{n}(\varphi \theta)^{2 n}\right\}-\left(b_{0} \varphi H+b_{1}(\varphi \theta)^{3}+\ldots+b_{n-2}(\varphi \theta)^{2 n-3}\right) \cdot f A \cdot F \theta}{\left(\varphi^{2} \theta-\varphi^{2} \theta_{1}\right)\left(\varphi^{2} \theta-\varphi^{2} H_{2}\right)\left(\varphi^{2} \theta-\varphi^{2} \theta_{3}\right) \ldots\left(\varphi^{2} \theta--\varphi^{2} H_{2 n}\right)}
$$

se réduit à une quantité finie pour $\theta=\theta_{1}, \theta_{2}, \ldots, \theta_{2 n}$ on aurn toujours:

$$
\varphi\left(\theta_{1}+\theta_{2}+\ldots+\theta_{2 n}\right)=0 \text {. }
$$

En faisant $\theta_{1}=\theta_{2}=\ldots=\theta_{2 n}=\alpha$ on en déduira un autre théorème:

Théorème IX. Si une équation de la formie

$$
\begin{gathered}
\left(a_{0}+a_{1} x^{2}+\ldots+a_{n} x^{2 n}\right)^{2} \\
-\left(b_{0} x+\ldots+b_{n-2} x^{2 n-3}\right)^{2}\left(1-c^{2} x^{2}\right)\left(1+e^{2} x^{2}\right)=a_{n}^{2}\left\{x^{2}-\varphi^{2} \alpha\right\}^{2 n}
\end{gathered}
$$

a lieu indépendemment de la valeur de $x$, la quantité $\alpha$ satisfera nécessairement à l'équation

$$
\varphi(2 n \alpha)=0
$$

c'est-à-dire on doit avoir $\alpha=\frac{m \omega+m^{\prime} \bar{\omega} i}{2 n}$ où $m$ et $m^{\prime}$ sont des nombres entiers. 
3. Si l'on fait

$$
\left\{\begin{array}{l}
p=a_{0} x+a_{1} x^{3}+\ldots+a_{n-1} x^{2 n-1} \\
q=b_{0}+b_{1} x^{2}+\ldots+b_{n-1} x^{2 n-2}
\end{array}\right.
$$

ou encore

$$
\left\{\begin{array}{l}
p=a_{0}+a_{1} x^{2}+\ldots+a_{n} x^{n} \\
q=b_{0} x+b_{2} x^{3}+\ldots+b_{n-1} x^{2 n-1}
\end{array}\right.
$$

on démontrera de la même manière les deux théorèmes suivants:

Théorème $\mathrm{X}$. Si l'expression

$$
\frac{a_{0} \varphi \theta+a_{1}(\varphi \theta)^{3}+\ldots+a_{n-1}(\varphi \theta)^{2 n-1}-\left(b_{0}+b_{1}(\varphi \theta)^{2}+\ldots+b_{n-1}(\varphi \theta)^{2 n-2}\right) \cdot f \theta \cdot F \theta}{\left(\varphi^{2} \theta-\varphi^{2} \theta_{1}\right)\left(\varphi^{2} \theta-\varphi^{2} \theta_{2}\right) \ldots\left(\varphi^{2} \theta-\varphi^{2} \theta_{2 n}\right)^{2}}
$$

reçoit une valeur finie pour $\theta=\theta_{1}, \theta_{2}, \ldots, \theta_{2 n}$ on aura toujours

$$
\varphi\left(\theta_{1}+\theta_{2}+\theta_{3}+\ldots+\theta_{2 n}\right)=\frac{\mathrm{I}}{\mathrm{o}} \text {. }
$$

Théorème XI. Si l'expression

$$
\frac{a_{0}+a_{1}(\varphi \theta)^{2}+\ldots+a_{n}(\varphi \theta)^{2 n}-\left(b_{0} \varphi \theta+b_{1}(\varphi \theta)^{3}+\ldots+b_{n-1}(\varphi \theta)^{2 n-1}\right) \cdot f \theta \cdot F \theta}{\left(\varphi^{2} \theta-\varphi^{2} \theta_{1}\right)\left(\varphi^{2} \theta-\varphi^{2} \theta_{2}\right) \ldots\left(\varphi^{2} \theta-\varphi^{2} \theta_{2 n+1}\right)}
$$

reçoit une valeur finie pour $\theta=\theta_{1}, \theta_{2}, \ldots, \theta_{2 n+1}$ on aura toujours

$$
\varphi\left(\theta_{1}+\theta_{2}+\ldots+\theta_{2 n+1}\right)=\frac{1}{0} .
$$

En supposant $\theta_{1}=\theta_{2}=\ldots=\alpha$, on aura comme corollaires:

Théorème XII. Si l'équation

$$
\begin{gathered}
\left(a_{0} x+a_{1} x^{3}+\ldots+a_{n-1} x^{2 n-1}\right)^{2} \\
-\left(b_{0}+b_{1} x^{2}+\ldots+b_{n-1} x^{2 n-2}\right)^{2}\left(\mathrm{I}-c^{2} x^{2}\right)\left(\mathrm{I}+e^{2} x^{2}\right)=c^{2} e^{2} b_{n-1}^{2}\left(x^{2}-\varphi^{2} \alpha\right)^{2 n+1}
\end{gathered}
$$

a lieu pour une valeur quelconque de $x$, la quantité a satisfera nécessairement à l'équation

$$
\varphi(2 n a)=\frac{1}{0}
$$


Théorème XIII. Si l'équation

$$
\begin{aligned}
\left(a_{0}+a_{1} x^{2}+\ldots+a_{n} x^{2 n}\right)^{2} & -\left(b_{0} x+b_{1} x^{3}+\ldots+b_{n-1} x^{2 n-1}\right)^{2}\left(\mathrm{I}-c^{2} x^{2}\right)\left(\mathrm{I}+e^{2} x^{2}\right) \\
& =c^{2} e^{2} b_{n-1}^{2}\left(x^{2}-\varphi^{2} \alpha\right)^{2 n+1}
\end{aligned}
$$

a lieu pour une valeur quelconque de $x$, la quantité $\alpha$ satisfera nécessairement à l'équation

$$
\varphi(2 n+1) \alpha=\frac{1}{0} .
$$

Ainsi p. ex. si l'on suppose $n=1$ on trouve que l'équation

$$
a_{0}^{2} x^{2}-b_{0}^{2}\left(\mathrm{1}-c^{2} x^{2}\right)\left(\mathrm{I}+e^{2} x^{2}\right)=e^{2} c^{2} b_{0}^{2}\left(x^{2}-\varphi^{2} \alpha\right)^{2}
$$

doit donner

$$
\varphi(2 a)=\frac{1}{0}
$$

L'équation précédente donne en effet $-\mathrm{I}=e^{2} c^{2} \varphi^{4} \alpha$ et cette relation entraîne l'équation $\varphi(2 \alpha)=\frac{1}{\mathrm{O}}$; comme on peut le voir par la formule

$$
\varphi 2 \alpha=\frac{2 \varphi \alpha \cdot f \alpha \cdot F \alpha}{1+e^{2} c^{2} \varphi^{4} \alpha} .
$$

\section{$\S 4$.}

Relations remarquables entre les quantités de la forme

$$
\varphi\left(\frac{m \omega+m^{\prime} \boldsymbol{\omega} i}{2 n+1}\right)
$$

La formule (38) du $\S 2$ donne pour la quantité $\varphi \varepsilon$ une valeur exprimée en fonction linéaire des quantités de la forme $\varphi(m \alpha+\mu \beta)$. Or on peut à l'aide d'un théorème démontré dans le paragraphe précédent trouver pour $\varphi \lesssim$ une autre expression beaucoup plus simple. En effet, en vertu du théorème VII l'équation (40) donnera nécessairement

d'où l'on tire

$$
\varphi(2 n+1) \varepsilon=0
$$

$$
\varepsilon=\frac{m \omega+\mu \sigma i}{2 n+\mathrm{I}}
$$


et par suite

$$
\varphi \varepsilon=\varphi\left(\frac{m \omega+\mu \bar{\omega} i}{2 n+1}\right)
$$

où $m$ et $\mu$ sont deux nombres entiers. Il en résulte en vertu de la formule (38) qu'on pourra toujours trouver deux nombres entiers $m$ et $\mu$ tels que

$$
\varphi\left(\frac{m \omega+\mu \bar{\omega} i}{2 n+1}\right)=\frac{1}{2 n+1} \cdot \sum_{0}^{2 n} \sum_{0}^{2 n} \partial^{m k+\mu k^{\prime}} \cdot \varphi(m \alpha+\mu \beta)
$$

quelles que soient d'ailleurs les valeurs des nombres entiers $k$ et $k^{\prime}$.

On aura en substituant les valeurs $\alpha=\frac{2 \omega}{2 n+\mathrm{I}}, \beta=\frac{2 \pi i}{2 n+\mathrm{I}}$

$$
\varphi\left(\frac{m \omega+\mu \bar{\omega} i}{2 n+1}\right)=\frac{1}{2 n+1} \cdot \sum_{0}^{2 n} \sum_{0}^{2 n} \partial^{m k+\mu k^{i}} \cdot \varphi\left(\frac{2 m \omega+2 \mu \bar{\omega} i}{216+1}\right) .
$$

Les nombres entiers $m$ et $\mu$ du prenier membre dépendront de la valeur de $k$ et $k^{\prime}$. Par un raisonnement, que je supprime ici, je suis parvenu à démontrer que $m=-(-1)^{n} \cdot n k^{\prime}, \mu=+(-1)^{n} k n$, en sorte qu'on aura pour des valeurs quelconques entières de $k, k^{\prime}$ et $n$

(IO2) $\quad \sum_{0}^{2 n} \sum_{0}^{2 n} \partial^{m k+\cdots k^{\prime}} \cdot \varphi\left(\begin{array}{c}2 m \omega+2 \mu \bar{\omega} i \\ 2 u+\mathrm{I}\end{array}\right)==-(-\mathrm{I})^{n} \cdot(2 n+\mathrm{I}) \cdot \varphi\left(\frac{n k^{\prime} \omega-n k \bar{\omega} i}{2 n+\mathrm{I}}\right)$

où

$$
\grave{j}=\cos \frac{2 \pi}{2 n+\mathrm{I}}+i \sin \frac{2 \pi}{2 n+\mathrm{I}} .
$$

On pourra déduire de la formule précédente plusieurs autres qui sont plus simples. Ainsi si l'on multiplie les deux membres par $\partial^{-4 t^{\prime}}$ et qu'on prenne ensuite la somme par rapport à $k^{\prime}$ depuis $k^{\prime}=0$ jusqu'à $k^{\prime}=2 n$ on trouvera

$$
\sum_{0}^{2 n} j^{m i k} \cdot \varphi\left(\frac{2 m \omega+2 n \bar{\omega} i}{2 n+\mathrm{I}}\right)=(-\mathrm{I})^{n+1} \cdot \sum_{0}^{2 n} j^{-\cdots k^{\prime}} \varphi\left(\frac{n k^{\prime} \omega-n k \bar{\omega} i}{2 n+\mathrm{I}}\right) .
$$

Si l'on fait $k=0$, on obtiendra en développant cette formule:

$$
\begin{aligned}
& \varphi\left(\frac{2 \nu \bar{\omega} i}{2 n+\mathrm{I}}\right)+\varphi\left(\frac{2 \omega+2 \nu \bar{\omega} i}{2 n+\mathrm{I}}\right)+\varphi\left(\frac{4 \omega+2 \nu \bar{\omega} i}{2 n+1}\right)+\ldots+\varphi\left(\frac{4 n \omega+2 \nu \bar{\omega} i}{2 n+\mathrm{I}}\right) \\
& =(-\mathrm{I})^{n+1} \cdot\left\{\delta^{-\nu \nu} \varphi\left(\frac{n \omega}{2 n+1}\right)+\delta^{-2 \nu} \varphi\left(\frac{2 n \omega}{2 n+1}\right)+\ldots+\partial^{-2 n \nu} \varphi\left(\frac{2 n^{2} \omega}{2 n+1}\right)\right\} .
\end{aligned}
$$


Recherches sur les fonctions elliptiques.

En multipliant les deux membres de l'équation (102) par $o^{-v k}$ et prenant ensuite la somme par rapport à $k$ depuis $k=0$ jusqu'à $k=2 n$, on obtiendra

$(105) \sum_{0}^{2 n} \partial^{y k^{\prime}} \cdot \varphi\left(\frac{2 \nu \omega+2 \mu \bar{\omega} i}{2 n+1}\right)=(-1)^{n+1} \cdot \sum_{0}^{2 n} j^{-\nu k} \cdot \varphi\left(\frac{n k^{\prime} \omega-n k \bar{\omega} i}{2 n+\mathrm{I}}\right)$.

Pour $k^{\prime}=0$ on obtiendra la formule

$$
\begin{aligned}
& \varphi\left(\frac{2 \nu \omega}{2 n+\mathrm{I}}\right)+\varphi\left(\frac{2 \nu \omega+2 \bar{\omega} i}{2 n+\mathrm{I}}\right)+\varphi\left(\frac{2 \nu \omega+4 \bar{\omega} i}{2 n+\mathrm{I}}\right)+\ldots+\varphi\left(\frac{2 \nu \omega+4 n \bar{\omega} i}{2 n+\mathrm{I}}\right) \\
= & (-\mathrm{I})^{n} \cdot\left\{{\partial^{-\nu}}^{-\nu}\left(\frac{n \bar{\omega} i}{2 n+\mathrm{I}}\right)+\partial^{-2 \nu} \varphi\left(\frac{2 n \bar{\omega} i}{2 n+\mathrm{I}}\right)+\ldots+\delta^{-2 n \nu} \varphi\left(\frac{2 n^{2} \bar{\omega} i}{2 n+\mathrm{I}}\right)\right\} .
\end{aligned}
$$

Si l'on suppose par ex. dans les deux formules (104), (I06) $n=\mathrm{I}, \nu=2$ on obtiendra:

$$
\begin{aligned}
& \varphi\left(\frac{4 \bar{\omega} i}{3}\right)+\varphi\left(\frac{2 \omega+4 \bar{\omega} i}{3}\right)+\varphi\left(\frac{4 \omega+4 \bar{\omega} i}{3}\right)=2 \sin \frac{2 \pi}{3} \cdot \varphi\left(\frac{\omega}{3}\right), \\
& \varphi\left(\frac{4 \omega}{3}\right)+\varphi\left(\frac{4 \omega+2 \pi i}{3}-\frac{4 \pi}{3}\right)+\varphi\left(\frac{4 \omega+4 \bar{\omega} i}{3}\right)=-2 \sin \frac{2 \pi}{3} \cdot \varphi\left(\frac{\bar{\omega} i}{3}\right)
\end{aligned}
$$

ou bien

$$
\begin{aligned}
& \varphi\left(\frac{\bar{\omega} i}{3}\right)-\varphi\left(\frac{\omega+\bar{\omega} i}{3}\right)+\varphi\left(\frac{\omega-\bar{\omega} i}{3}\right)=-\sqrt{3} \cdot i \cdot \varphi\left(\frac{\omega}{3}\right) \\
& \varphi\left(\frac{\omega}{3}\right)-\varphi\left(\frac{\omega+\bar{\omega} i}{3}\right)-\varphi\left(\frac{\omega-\bar{\omega} i}{3}\right)=+\sqrt{3} \cdot i \cdot \varphi\left(\frac{\bar{\omega} i}{3}\right)
\end{aligned}
$$

on en tire

$$
\left\{\begin{array}{l}
\varphi\left(\frac{\omega+\bar{\omega} i}{3}\right)=\frac{\mathrm{I}+i \sqrt{3}}{2} \varphi\left(\frac{\omega}{3}\right)+\frac{\mathrm{I}-i \sqrt{3}}{2} \cdot \varphi\left(\frac{\bar{\omega} i}{3}\right), \\
\varphi\left(\frac{\omega-\bar{\omega} i}{3}\right)=\frac{\mathrm{I}-i \sqrt{3}}{2} \varphi\left(\frac{\omega}{3}\right)-\frac{\mathrm{1}+i \sqrt{3}}{2} \cdot \varphi\left(\frac{\bar{\omega} i}{3}\right) .
\end{array}\right.
$$

On pourra encore trouver d'autres relations plus simples que celles exprimćes par les formules (104), (106). On a en effet:

$$
\begin{aligned}
& (\mathrm{I} \circ 8) \quad \mathrm{O}=\varphi\left(\frac{m \bar{\omega} i}{2 n+\mathrm{I}}\right)-\delta^{m} \varphi\left(\frac{\omega+m \bar{\omega} i}{2 n+\mathrm{I}}\right)+\delta^{-m} \varphi\left(\frac{\omega-m \bar{\omega} i}{2 n+\mathrm{I}}\right)+\delta^{2 m} \varphi\left(\frac{2 \omega+m \bar{\omega} i}{2 n+\mathrm{I}}\right) \\
& -\delta^{-2 m} \varphi\left(\frac{2 \omega-m \bar{\omega} i}{2 n+\mathrm{I}}\right)-\ldots+(-\mathrm{I})^{n}\left\{\delta^{n m} \varphi\left(\frac{n \omega+m \bar{\omega} i}{2 n+\mathrm{I}}\right)-\delta^{-n m} \varphi\left(\frac{n \omega-m \bar{\omega} i}{2 n+\mathrm{I}}\right)\right\}
\end{aligned}
$$




$$
\begin{aligned}
& (\mathrm{I} 09) \mathrm{O}=\varphi\left(\frac{m \omega}{2 n+\mathrm{I}}\right)+\delta^{m} \varphi\left(\frac{m \omega-\bar{\omega} i}{2 n+\mathrm{I}}\right)+\delta^{-m} \varphi\left(\frac{m \omega+\bar{\omega} i}{2 n+\mathrm{I}}\right)-\delta^{2 m} \varphi\left(\frac{m \omega-2 \bar{\omega} i}{2 n+\mathrm{I}}\right) \\
& -\delta^{-2 m} \varphi\left(\frac{m \omega-2 \bar{\omega} i}{2 n+1}\right)+\ldots-(-\mathrm{I})^{n} \cdot\left\{\delta^{m n} \varphi\left(\frac{m \omega-n \bar{\sigma} i}{2 n+\mathrm{I}}\right)+\delta^{-m n} \varphi\left(\frac{m \omega+n \bar{\omega} i}{2 n+\mathrm{I}}\right)\right\} .
\end{aligned}
$$

Ces équations peuvent remplacer la formule (102) dans toute sa généralité. Elles donneront des résultats différents pour $m=0,1,2,3, \ldots, n$, mais ceux qu'on obtiendra pour une autre valeur de $m$ rentreront dans ceux-là.

\section{$\$ 5$.}

Je terminerai ce second mémoire par l'énoncé de plusieurs théorèmes qui me paraissent de quelque importance.

Théorème XIV. Soient pour abréger $\alpha=\frac{2 m \omega+2 \mu \tilde{\omega} i}{2 n+1}$ où les trois nombres entiers $m, \mu, 2 n+\mathrm{I}$ ne sont pas divisibles par le même facteur et

$$
\begin{gathered}
y=\varphi(\theta) \cdot \varphi(\alpha-\theta) \cdot \varphi(\alpha+\theta) \cdot \varphi(2 \alpha-\theta) \cdot \varphi(2 \alpha+\theta) \ldots \varphi(n \alpha-\theta) \cdot \varphi(n \alpha+\theta), \\
c_{1}=c^{2 n+1} \cdot \frac{\mathrm{I}+e^{2} \varphi^{2} \alpha}{\mathrm{I}-c^{2} \varphi^{2} \alpha} \cdot \frac{\mathrm{I}+e^{2} \varphi^{2} 2 \alpha}{\mathrm{I}-c^{2} \varphi^{2} 2 \alpha} \cdots \frac{\mathrm{I}+e^{2} \varphi^{2} n \alpha}{\mathrm{I}-c^{2} \varphi^{2} n \alpha}, \\
e_{1}=e^{2 n+1} \cdot \frac{\mathrm{I}-c^{2} \varphi^{2} \alpha}{\mathrm{I}+e^{2} \varphi^{2} \alpha} \cdot \frac{\mathrm{I}-c^{2} \varphi^{2} 2 \alpha}{\mathrm{I}+e^{2} \varphi^{2} 2 \alpha} \cdots \frac{\mathrm{I}-c^{2} \varphi^{2} n \alpha}{\mathrm{I}+e^{2} \varphi^{2} n \alpha},
\end{gathered}
$$

Cela posé, si l'on désigne par $P$ une fonction quelconque entière des $2 n$ quantités:

$$
\varphi \theta, \varphi(\theta+\alpha), \varphi(\theta+2 a), \ldots, \varphi(\theta+2 n a)
$$

qui aura la propriété de rester invariable par le changement de $\theta$ en $\theta+\alpha$; on pourra toujours faire

$$
P=p+q \cdot 1 \overline{\left(1-c_{1}^{2} y^{2}\right)\left(\mathrm{I}+e_{1}^{2} y^{2}\right)}
$$

où $p$ et $q$ sont deux fonctions entières de la quantité $y$. Si l'on désigne par $P^{\prime}$ la valeur de $P$ lorsqu'on y change le signe de $\alpha$ on aura en même temps

$$
P^{\prime}=p-q \cdot \sqrt{\left(\mathbf{1}-c_{1}^{2} y^{q}\right)\left(\mathbf{I}+e_{1}^{\mathbf{a}} y^{\mathbf{2}}\right)} \text {. }
$$

En désignant par $\nu$ le degré de la fonction proposée $P$ par rapport à $\varphi \theta$, 
les fonctions $p$ et $q$ seront respectivement des degrés $\nu$ et $\nu-2$ par rapport ̀̀ $y$.

Ia démonstration de ce théorème est principalement fondée sur cela que les racines de l'équation

$$
\begin{gathered}
\mathrm{O}=x\left(\varphi^{2} \alpha-x^{2}\right)\left(\varphi^{2} 2 \alpha-x^{2}\right) \ldots\left(\varphi^{2} n \alpha-x^{2}\right) \\
-y\left(\mathrm{I}+e^{2} c^{2} \varphi^{2} \alpha \cdot x^{2}\right)\left(\mathrm{I}+e^{2} c^{2} \varphi^{2} 2 \alpha \cdot x^{2}\right) \ldots\left(\mathrm{I}+e^{2} c^{2} \varphi^{2} n \alpha \cdot x^{2}\right)
\end{gathered}
$$

sont les $2 n+\mathrm{I}$ quantités

$$
\varphi \theta, \varphi(\theta+a), \ldots, \varphi(\theta+2 n \alpha) \text {. }
$$

Si l'on pose par ex.:

$$
P=\varphi \theta+\varphi(\theta+\alpha)+\ldots+\varphi(\theta+2 n \alpha)
$$

on trouvera cette formule:

$$
\begin{gathered}
(-1)^{n} \cdot \varphi \theta \cdot \varphi(\alpha-\theta) \cdot \varphi(\alpha+\theta) \ldots \varphi(n \alpha+\theta) \cdot \varphi(n \alpha-\theta) \cdot e^{2 n} \cdot c^{2 n} \cdot(\varphi \alpha \cdot \varphi 2 \alpha \ldots \varphi n \alpha)^{2} \\
=\varphi \theta+\varphi(\theta+\alpha)+\varphi(\theta+2 \alpha)+\ldots+\varphi(\theta+2 n \alpha) .
\end{gathered}
$$

Si l'on fait:

$$
P=\left\{\varphi \theta+\delta^{\mu} \varphi(\theta+\alpha)+\delta^{2 \mu} \varphi(\theta+2 x)+\ldots+\delta^{2 n \mu} \varphi(\theta+2 n \alpha)\right\}^{2 n+1}
$$

où

$$
\grave{o}=\cos \frac{2 \pi}{2 n+1}+i \sin \frac{2 \pi}{2 n+1}
$$

$P$ aura la propriété requise et l'on trouvera:

$$
\begin{gathered}
P=a_{0} y+a_{1} y^{2}+a_{2} y^{3}+\ldots+a_{n} y^{2 n+1} \\
+\left(b_{0}+b_{1} y^{2}+\ldots+b_{n-1} y^{2 n-2}\right) \sqrt{\left(\mathbf{I}-c_{1}^{2} y^{2}\right)\left(\mathrm{r}+e_{1}^{2} y^{2}\right)}
\end{gathered}
$$

où les coefficients $a_{0}, a_{1}, \ldots, b_{0}, b_{1}, \ldots, b_{n-1}$ satisfont ̀̀ l'équation

$$
\begin{gathered}
\left(a_{0} y+a_{1} y^{3}+\cdots+a_{n} y^{2 n+1}\right)^{2}-\left(b_{0}+b_{1} y^{2}+\cdots+b_{n-1} y^{2 n-2}\right)^{2}\left(\mathrm{I}-c_{1}^{2} y^{2}\right)\left(\mathrm{I}+e_{1}^{2} y^{2}\right) \\
=a_{n}^{2}\left(y^{2}-k^{2}\right)^{2 n+1}
\end{gathered}
$$

pour une valeur quelconque $y$. 
Connaissant la valeur de $P$ pour chaque valeur de $\mu$ il est facile d'en tirer la résolution algébrique de l'équation. On a en effet en désignant par $P_{n}$ la valeur de $P$ qui répond à $\mu$ :

$$
\varphi \theta+\delta^{\mu} \varphi(\theta+\alpha)+\delta^{2 \mu} \varphi(\theta+2 \alpha)+\ldots+\delta^{2 n n^{\prime}} \varphi(\theta+2 n \alpha)=\sqrt[2 n+1]{P_{\mu}}
$$

d'où l'on tire ensuite en faisant $\mu=0,1,2, \ldots, 2 n$

$$
\begin{gathered}
\varphi(\theta+m \alpha)=\frac{\mathrm{I}}{2 n+\mathrm{I}}\left\{(-\mathrm{I})^{n} e^{2 n} c^{2 n}(\varphi \alpha \cdot \varphi 2 \alpha \ldots \varphi n \alpha)^{2} \cdot y\right. \\
\left.+\delta^{-\mu} \cdot \sqrt[2 n+1]{P_{1}}+\delta^{-2 n} \cdot \sqrt[2 n+1]{P_{2}}+\ldots+\delta^{-2 n / t} \cdot \sqrt[2 n+1]{P_{2 n}}\right\}
\end{gathered}
$$

ce qui est l'expression d'une racine quelconque de l'équation.

Théorème XV. IA quantité $\varphi\left(\frac{m \bar{\omega} i+k \omega}{2 n+I}\right)$ pourra toujours s'exprimer algébriquement en fonction de $\varphi\left(\frac{\omega}{2 n+1}\right)$. On aura en effet

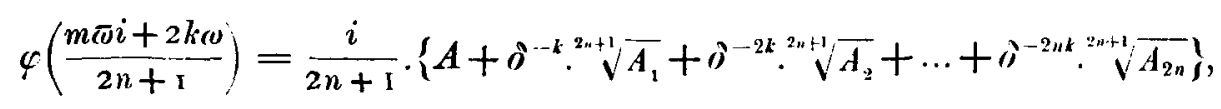

où $A, A_{1}, \ldots, A_{2 n}$ sont des quantités réelles et en même temps des fonetions rationnelles des deux quantités $c\left(\frac{\omega}{2 n+1}\right)$ et $\sin \left(\frac{\pi}{2 n+1}\right)$. Pour $k=0$ on aura

$$
\varphi\left(\frac{m \tilde{\boldsymbol{\sigma}} i}{2 n+\mathrm{I}}\right)=\frac{i}{2 n+\mathrm{I}} \cdot\left\{A+\sqrt[2 n+1]{A_{1}}+\sqrt[2 n+1]{A_{z}}+\ldots+\sqrt[2 n+1]{\sqrt[A_{2 n}]{3}}\right\} .
$$

Il est à remarquer que l'une des quantités $A_{1}, A_{2}, \ldots, A_{2 n}$ est nécessairement égale à zéro. Cela se voit à l'aide de la formule ( IO8).

La démonstration de ce théorème se déduit des deux formules ( I 6 ), (104).

Théorème XVI. Si les deux quantités $\omega$ et $\overline{\boldsymbol{D}}$ sont liées entre elles par l'équation $\omega=\bar{\omega} \cdot \sqrt{2 n+\mathrm{I}}$, c'est-à-dire si l'on a

$$
\int_{0}^{\frac{1}{c}} \frac{d x}{\sqrt{\left(\mathrm{I}-c^{2} x^{2}\right)\left(\mathrm{I}+e^{2} x^{2}\right)}}=\sqrt{2 n+1} \int_{0}^{\frac{1}{e}} \frac{d x}{\sqrt{\left(1+c^{2} x^{2}\right)\left(\mathrm{I}-e^{2} x^{2}\right)}}
$$


où $e$ et $c$ sont des quantités réelles et positives, alors la fonction $\varphi$ aura la propriété suivante:

$$
\begin{gathered}
\sin \left(\frac{2 \mu \pi}{2 n+1}\right) \cdot \varphi\left(\frac{\omega}{2 n+\mathrm{I}}\right)-\sin \left(\frac{4 \mu \pi}{2 n+\mathrm{I}}\right) \cdot \varphi\left(\frac{2 \omega}{2 n+\mathrm{I}}\right)+\ldots \\
-(-\mathrm{I})^{n} \sin \left(\frac{2 n \mu \pi}{2 n+\mathrm{I}}\right) \cdot \varphi\left(\frac{n \omega}{2 n+\mathrm{I}}\right)=(-\mathrm{I})^{n+\mu} \cdot \sqrt{2 n+1} \cdot \varphi\left(\frac{\mu \omega}{2 n+\mathrm{I}}\right),
\end{gathered}
$$

où $\mu$ désigne un nombre entier quelconque.

Si par ex. $n=2, \mu=1$ on aura $\omega=\bar{\omega} \sqrt{5}$ et

$$
\sin \left(\frac{2 \pi}{5}\right) \cdot \varphi\left(\frac{\omega}{5}\right)-\sin \left(\frac{\pi}{5}\right) \cdot \varphi\left(\frac{2 \omega}{5}\right)=-\sqrt{5} \cdot \varphi\left(\frac{\omega}{5}\right) .
$$

Fin du second mémoire.

Christiania le 27 août 1828 . 